\title{
Well-posedness of history-dependent evolution inclusions with applications
}

\author{
Stanisław Migórski and Yunru Bai
}

\begin{abstract}
In this paper, we study a class of evolution subdifferential inclusions involving history-dependent operators. We improve our previous theorems on existence and uniqueness and produce a continuous dependence result with respect to weak topologies under a weaker smallness condition. Two applications are provided to a frictional viscoelastic contact problem with long memory, and to a nonsmooth semipermeability problem.
\end{abstract}

Mathematics Subject Classification. 47J20, 47J22, 49J40, 49J45, 74G25, 74G30, 74M15.

Keywords. Evolution inclusion, Variational-hemivariational inequality, Clarke subdifferential, History-dependent operator, Existence and uniqueness, Frictional contact, Semipermeability.

\section{Introduction}

In this paper, we study a class of the Cauchy problems for evolution inclusions of subdifferential type in the framework of evolution triple of spaces. Recently, the problem has been studied in [21] without the convex subdifferential term and under the sign condition for the nonconvex potential, and in [8], where reformulated as a variational-hemivariational inequality, has been analyzed under a restrictive smallness hypothesis on the constants involved in operators $A$ and $\partial J$ [see problem (1.1), (1.2) below]. Here, we replace this restriction by a weaker condition and further examine the continuous dependence for such inclusions which was not studied before. In this way, the present paper is a continuation of [8]. Moreover, we provide a new application of our results to a semipermeability problem with nonmonotone and possibly multivalued subdifferential boundary conditions.

The main feature of the evolution inclusion under consideration is that both multivalued terms are generated by subdifferential operators which take their values in the dual space and not in a pivot space, and moreover, such inclusions depend on operators involved in the subdifferential maps and assumed to be history-dependent. We should note that Gasinski et al. [6] investigated an abstract first-order evolution inclusion in a reflexive Banach space extending several earlier work on parabolic hemivariational inequalities by Migórski [13], and Migórski and Ochal [15], and others.

The initial value problem for evolution inclusion under consideration reads as follows. Find $w \in$ $L^{2}(0, T ; V)$ with $w^{\prime} \in L^{2}\left(0, T ; V^{*}\right)$ such that

$$
\begin{aligned}
& w^{\prime}(t)+A(t, w(t))+\left(R_{1} w\right)(t)+M^{*} \partial J(t,(S w)(t), M w(t)) \\
& \quad+N^{*} \partial \varphi(t,(R w)(t), N w(t)) \ni f(t) \text { a.e. } t \in(0, T), \\
& w(0)=w_{0} .
\end{aligned}
$$

Project supported by the H2020-MSCA-RISE-2018 Research and Innovation Staff Exchange Scheme Fellowship within the Project No. 823731 CONMECH, the NSF of Guangxi Grant No. 2018JJA110006, and Beibu Gulf University Project No. 2018KYQD06. 
In inclusion (1.1), the symbol $\partial J$ stands for the generalized gradient of a locally Lipschitz function $J(t, z, \cdot)$ and $\partial \varphi$ denotes the convex subdifferential of a convex and lower semicontinuous function $\varphi(t, y, \cdot)$. Furthermore, (1.1) involves three nonlinear operators $R, R_{1}$ and $S$ assumed to be history-dependent. It is worth to observe that inclusion (1.1) finds an equivalent formulation as the following history-dependent variational-hemivariational inequality, see [8, Problem 7]:

$$
\begin{aligned}
& \left\langle w^{\prime}(t)+A(t, w(t))+\left(R_{1} w\right)(t)-f(t), v-w(t)\right\rangle_{V^{*} \times V} \\
& \quad+J^{0}(t,(S w)(t), w(t) ; v-w(t))+\varphi(t,(R w)(t), v)-\varphi(t,(R w)(t), w(t)) \geq 0
\end{aligned}
$$

for all $v \in V$, a.e. $t \in(0, T)$. Existence and uniqueness results for a particular form of inclusion (1.3), under more restrictive hypotheses, have been recently proved in [22] where $J \equiv 0$, and in [23] where $J$ is independent of the history-dependent operator. We refer to [14,17, 18,20,28,29,31,33] for various related results on history-dependent inequality problems, and to a recent monograph [32] for a comprehensive research. Moreover, various classes of related differential variational inequalities and differential hemivariational inequalities have been studied only recently in [10-12,24,35].

The novelties of the paper are following. First, we prove existence of the unique solution to problem (1.1), (1.2) under a weaker (relaxed) smallness condition than in [8, Theorem 9]. Since the smallness condition is simpler here, it is applied to a wider class of evolution inclusions. Second, we deliver a continuous dependence result for the map $\left(f, w_{0}\right) \mapsto w$ for problem (1.1), (1.2) in weak topologies. Note that a convergence result in norm topologies for a problem in which $\partial J$ does not depend on the history-dependent operator was obtained in [32, Theorem 99]. Third, we provide a new application of the continuous dependence result of Theorem 7 to a dynamic frictional contact model with history-dependent operators, and a new well-posedness result for a semipermeability problem. Finally, note that the continuous dependence result in weak topologies obtained in this paper can be used in analysis of various optimization and optimal control problems for variational and variational-hemivariational inequalities with history-dependent operators.

\section{Essential tools}

Let us recall some basic definitions from nonlinear analysis in Banach spaces. For more details in this connection, we refer to, e.g., [2-4].

Let $X$ be a Banach space. Throughout this paper, we denote by $\langle\cdot, \cdot\rangle_{X{ }^{*} \times X}$ the duality pairing between a Banach space $X$ and its dual $X^{*}$, and by $\|\cdot\|_{X}$ the norm in $X$. When no confusion arises, we often drop the subscripts. A function $\varphi: X \rightarrow \mathbb{R} \cup\{+\infty\}$ is proper if its effective domain $\operatorname{dom} \varphi=\{x \in X \mid$ $\varphi(x)<+\infty\} \neq \emptyset$. It is lower semicontinuous (l.s.c.) if $x_{n} \rightarrow x$ in $X$ entails $\varphi(x) \leq \lim \inf \varphi\left(x_{n}\right)$.

Let $\varphi: X \rightarrow \mathbb{R} \cup\{+\infty\}$ be a convex function. An element $x^{*} \in X^{*}$ is called a subgradient of $\varphi$ at $u \in X$ if

$$
\left\langle x^{*}, v-u\right\rangle_{X^{*} \times X} \leq \varphi(v)-\varphi(u) \text { for all } v \in X .
$$

The set of all $x^{*} \in X^{*}$ which satisfy (2.1) is called the (convex) subdifferential of $\varphi$ at $u$ and is denoted by $\partial \varphi(u)$. Next, we recall the notions of the generalized directional derivative and the generalized gradient of Clarke for a locally Lipschitz function $\psi: X \rightarrow \mathbb{R}$. The generalized directional derivative of $\psi$ at $u \in X$ in the direction $v \in X$ is defined by

$$
\psi^{0}(u ; v)=\limsup _{y \rightarrow u, t \downarrow 0} \frac{\psi(y+t v)-\psi(y)}{t} .
$$

The generalized gradient of $\psi$ at $u \in X$ is given by

$$
\partial \psi(u)=\left\{u^{*} \in X^{*} \mid \psi^{0}(u ; v) \geq\left\langle u^{*}, v\right\rangle_{X^{*} \times X} \text { for all } v \in X\right\} .
$$


The locally Lipschitz function $\psi$ is called regular (in the Clarke sense) at $u \in X$ if for all $v \in X$ the one-sided directional derivative $\psi^{\prime}(u ; v)$ exists and satisfies $\psi^{0}(u ; v)=\psi^{\prime}(u ; v)$ for all $v \in X$. In what follows the generalized gradient of Clarke for a locally Lipschitz function and the subdifferential of a convex function will be denoted in the same way.

Recall that an operator $A: X \rightarrow X^{*}$ is said to be demicontinuous if for all $v \in X$, the functional $u \mapsto\langle A u, v\rangle_{X^{*} \times X}$ is continuous, i.e., $A$ is continuous as a map from $X$ to $X^{*}$ endowed with the weak topology. Let $0<T<\infty$ and $A:(0, T) \times X \rightarrow X^{*}$. The Nemytskii (superposition) operator associated with $A$ is the operator $\mathcal{A}: L^{2}(0, T ; X) \rightarrow L^{2}\left(0, T ; X^{*}\right)$ defined by

$$
(\mathcal{A} v)(t)=A(t, v(t)) \text { for } v \in L^{2}(0, T ; X) \text {, a.e. } t \in(0, T) .
$$

A multivalued operator $A: X \rightarrow 2^{X^{*}}$ is called coercive if either its domain $D(A)=\{u \in X \mid A u \neq \emptyset\}$ is bounded or $D(A)$ is unbounded and

$$
\lim _{\|u\|_{X} \rightarrow \infty, u \in D(A)} \frac{\inf \left\{\left\langle u^{*}, u\right\rangle_{X^{*} \times X} \mid u^{*} \in A u\right\}}{\|u\|_{X}}=+\infty .
$$

Given a set $D$ in a normed space $E$, we define $\|D\|_{E}=\sup \left\{\|x\|_{E} \mid x \in D\right\}$. The space of linear and bounded operators from a normed space $E$ to a normed space $F$ is denoted by $\mathcal{L}(E, F)$. It is endowed with the standard operator norm $\|\cdot\|_{\mathcal{L}(E, F)}$. For an operator $L \in \mathcal{L}(E, F)$, we denote its adjoint by $L^{*} \in \mathcal{L}\left(F^{*}, E^{*}\right)$.

Recall that the spaces $\left(V, H, V^{*}\right)$ form an evolution triple of spaces, if $V$ is a reflexive and separable Banach space, $H$ is a separable Hilbert space, and the embedding $V \subset H$ is dense and continuous. We introduce the following Bochner spaces $\mathcal{V}=L^{2}(0, T ; V), \mathcal{V}^{*}=L^{2}\left(0, T ; V^{*}\right)$, and $\mathcal{W}=\left\{w \in \mathcal{V} \mid w^{\prime} \in \mathcal{V}^{*}\right\}$. It follows from standard results, see, e.g., [3, Section 8.4], that the space $\mathcal{W}$ endowed with the graph norm $\|w\|_{\mathcal{W}}=\|w\|_{\mathcal{V}}+\left\|w^{\prime}\right\|_{\mathcal{V}^{*}}$ is a separable and reflexive Banach space, and each element in $\mathcal{W}$, after a modification on a set of null measure, can be identified with a unique continuous function on $[0, T]$ with values in $H$. Further, the embedding $\mathcal{W} \subset C(0, T ; H)$ is continuous, where $C(0, T ; H)$ stands for the space of continuous functions on $[0, T]$ with values in $H$.

Finally, we state a fixed point result (see [9, Lemma 7] or [30, Proposition 3.1]) being a consequence of the Banach contraction principle.

Lemma 1. Let $X$ be a Banach space and $0<T<\infty$. Let $F: L^{2}(0, T ; X) \rightarrow L^{2}(0, T ; X)$ be an operator such that

$$
\left\|\left(F v_{1}\right)(t)-\left(F v_{2}\right)(t)\right\|_{X}^{2} \leq c \int_{0}^{t}\left\|v_{1}(s)-v_{2}(s)\right\|_{X}^{2} \mathrm{~d} s
$$

for all $v_{1}, v_{2} \in L^{2}(0, T ; X)$, a.e. $t \in(0, T)$ with a constant $c>0$. Then, there exists a unique $v^{*} \in$ $L^{2}(0, T ; X)$ such that $F v^{*}=v^{*}$.

\section{History-dependent evolution inclusions}

We start with the study of existence and uniqueness for abstract first-order evolution subdifferential inclusion in a general form. Our study is a continuation of paper [8] where a class of general dynamic history-dependent variational-hemivariational inequalities has been investigated. The aim is to provide an improved version of result in [8, Theorem 6] which actually holds under a more general smallness hypothesis.

We study the operator inclusions in the standard functional setting used for evolution problems which exploits the notion of an evolution triple of spaces $\left(V, H, V^{*}\right)$. We use the notation $\mathcal{V}, \mathcal{V}^{*}$ and $\mathcal{W}$, recalled in the previous section.

Given $A:(0, T) \times V \rightarrow V^{*}, \psi:(0, T) \times V \rightarrow \mathbb{R}, f:(0, T) \rightarrow V^{*}$ and $w_{0} \in V$, we consider the following Cauchy problem for the evolution inclusion. 
Problem 2. Find $w \in \mathcal{W}$ such that

$$
\left\{\begin{array}{l}
w^{\prime}(t)+A(t, w(t))+\partial \psi(t, w(t)) \ni f(t) \text { a.e. } t \in(0, T), \\
w(0)=w_{0} .
\end{array}\right.
$$

Here, $\psi(t, \cdot)$ is a locally Lipschitz function and $\partial \psi$ denotes its Clarke generalized gradient. We recall that a function $w \in \mathcal{W}$ is a solution of Problem 2, if there exists $w^{*} \in \mathcal{V}^{*}$ such that $w^{\prime}(t)+A(t, w(t))+$ $w^{*}(t)=f(t)$ a.e. $t \in(0, T), w^{*}(t) \in \partial \psi(t, w(t))$ a.e. $t \in(0, T)$, and $w(0)=w_{0}$.

In the study of Problem 2, we need the following hypotheses.

$H(A): A:(0, T) \times V \rightarrow V^{*}$ is such that

(i) $A(\cdot, v)$ is measurable on $(0, T)$ for all $v \in V$.

(ii) $A(t, \cdot)$ is demicontinuous on $V$ for a.e. $t \in(0, T)$.

(iii) $\|A(t, v)\|_{V^{*}} \leq a_{0}(t)+a_{1}\|v\|_{V}$ for all $v \in V$, a.e. $t \in(0, T)$ with $a_{0} \in L^{2}(0, T), a_{0} \geq 0$ and $a_{1} \geq 0$.

(iv) $A(t, \cdot)$ is strongly monotone for a.e. $t \in(0, T)$, i.e., for a constant $m_{A}>0$,

$$
\left\langle A\left(t, v_{1}\right)-A\left(t, v_{2}\right), v_{1}-v_{2}\right\rangle_{V^{*} \times V} \geq m_{A}\left\|v_{1}-v_{2}\right\|_{V}^{2}
$$

for all $v_{1}, v_{2} \in V$, a.e. $t \in(0, T)$.

$H(\psi): \psi:(0, T) \times V \rightarrow \mathbb{R}$ is such that

(i) $\psi(\cdot, v)$ is measurable on $(0, T)$ for all $v \in V$.

(ii) $\psi(t, \cdot)$ is locally Lipschitz on $V$ for a.e. $t \in(0, T)$.

(iii) $\|\partial \psi(t, v)\|_{V^{*}} \leq c_{0}(t)+c_{1}\|v\|_{V}$ for all $v \in V$, a.e. $t \in(0, T)$ with $c_{0} \in L^{2}(0, T), c_{0} \geq 0, c_{1} \geq 0$.

(iv) $\partial \psi(t, \cdot)$ is relaxed monotone for a.e. $t \in(0, T)$, i.e., for a constant $m_{\psi} \geq 0$,

$$
\left\langle z_{1}-z_{2}, v_{1}-v_{2}\right\rangle_{V^{*} \times V} \geq-m_{\psi}\left\|v_{1}-v_{2}\right\|_{V}^{2}
$$

for all $z_{i} \in \partial \psi\left(t, v_{i}\right), z_{i} \in V^{*}, v_{i} \in V, i=1$, 2, a.e. $t \in(0, T)$.

$\left(H_{0}\right): f \in \mathcal{V}^{*}, w_{0} \in V$.

$\overline{\left(H_{1}\right)}: m_{A}>m_{\psi}$.

We have the following existence and uniqueness result.

Theorem 3. Under hypotheses $H(A), H(\psi),\left(H_{0}\right)$ and $\left(H_{1}\right)$, Problem 2 has a unique solution.

Proof. The proof follows the lines of [8, Theorem 6]. For this reason, we provide only an argument for the coercivity of the operator $\mathcal{F}: \mathcal{V} \rightarrow 2^{\mathcal{V}^{*}}$ defined by $\mathcal{F} v=\mathcal{A} v+\mathcal{B} v$ for $v \in \mathcal{V}$, where $\mathcal{A}: \mathcal{V} \rightarrow \mathcal{V}^{*}$ and $\mathcal{B}: \mathcal{V} \rightarrow 2^{\mathcal{V}^{*}}$ are the Nemytskii operators corresponding to the translations of $A(t, \cdot)$ and $\partial \psi(t, \cdot)$ by the initial condition $w_{0}$ :

$$
\begin{aligned}
& (\mathcal{A} v)(t)=A\left(t, v(t)+w_{0}\right), \\
& \mathcal{B} v=\left\{v^{*} \in \mathcal{V}^{*} \mid v^{*}(t) \in \partial \psi\left(t, v(t)+w_{0}\right) \text { for a.e. } t \in(0, T)\right\}
\end{aligned}
$$

for $v \in \mathcal{V}$ and a.e. $t \in(0, T)$. By $H(A)$ (iii), (iv) and $H(\psi)$ (iii), (iv), we have

$$
\begin{aligned}
& \langle A(t, z)+\partial \psi(t, z), z\rangle_{V^{*} \times V}=\langle A(t, z)-A(t, 0), z\rangle_{V^{*} \times V} \\
& \quad+\langle\partial \psi(t, z)-\partial \psi(t, 0), z\rangle_{V^{*} \times V}+\langle A(t, 0)+\partial \psi(t, 0), z\rangle_{V^{*} \times V} \\
& \quad \geq\left(m_{A}-m_{\psi}\right)\|z\|_{V}^{2}-\left(a_{0}(t)+c_{0}(t)\right)\|z\|_{V}
\end{aligned}
$$

for all $z \in V$, a.e. $t \in(0, T)$. Next, let $v \in \mathcal{V}$. We obtain

$$
\begin{aligned}
\langle\mathcal{A} v & +\mathcal{B} v, v\rangle_{\mathcal{V}^{*} \times \mathcal{V}}=\int_{0}^{T}\langle(\mathcal{A} v)(t)+(\mathcal{B} v)(t), v(t)\rangle_{V^{*} \times V} \mathrm{~d} t \\
= & \int_{0}^{T}\left\langle A\left(t, v(t)+w_{0}\right)+\partial \psi\left(t, v(t)+w_{0}\right), v(t)+w_{0}\right\rangle_{V^{*} \times V} \mathrm{~d} t \\
& -\int_{0}^{T}\left\langle A\left(t, v(t)+w_{0}\right)+\partial \psi\left(t, v(t)+w_{0}\right), w_{0}\right\rangle_{V^{*} \times V} \mathrm{~d} t .
\end{aligned}
$$


Using $H(A)$ (iii) and $H(\psi)($ iii), we get

$$
\begin{aligned}
& \left|\left\langle A\left(t, v(t)+w_{0}\right)+\partial \psi\left(t, v(t)+w_{0}\right), w_{0}\right\rangle_{V^{*} \times V}\right| \\
& \quad \leq\left(\left\|A\left(t, v(t)+w_{0}\right)\right\|_{V^{*}}+\left\|\partial \psi\left(t, v(t)+w_{0}\right)\right\|_{V^{*}}\right)\left\|w_{0}\right\|_{V} \\
& \quad \leq\left(a_{0}(t)+c_{0}(t)+\left(a_{1}+c_{1}\right)\left\|w_{0}\right\|_{V}\right)\left\|w_{0}\right\|_{V}+\left(a_{1}+c_{1}\right)\left\|w_{0}\right\|_{V}\|v(t)\|_{V} .
\end{aligned}
$$

Integrating this inequality on $(0, T)$, by the Hölder inequality, we deduce

$$
\begin{aligned}
& -\int_{0}^{T}\left\langle A\left(t, v(t)+w_{0}\right)+\partial \psi\left(t, v(t)+w_{0}\right), w_{0}\right\rangle_{V^{*} \times V} \mathrm{~d} t \geq-\left(a_{1}+c_{1}\right)\left\|w_{0}\right\|_{V}^{2} \\
& \quad-\sqrt{T}\left(\left\|a_{0}\right\|_{L^{2}}+\left\|c_{0}\right\|_{L^{2}}\right)\left\|w_{0}\right\|_{V}-\sqrt{T}\left(a_{1}+c_{1}\right)\left\|w_{0}\right\|_{V}\|v\|_{\mathcal{V}} .
\end{aligned}
$$

Combining (3.1) and (3.2), we obtain

$$
\begin{aligned}
& \langle\mathcal{A} v+\mathcal{B} v, v\rangle_{\mathcal{V}^{*} \times \mathcal{V}} \geq\left(m_{A}-m_{\psi}\right)\left\|v+w_{0}\right\|_{\mathcal{V}}^{2}-\left(\left\|a_{0}\right\|_{L^{2}}+\left\|c_{0}\right\|_{L^{2}}\right)\left\|v+w_{0}\right\|_{\mathcal{V}} \\
& \quad-\sqrt{T}\left(\left\|a_{0}\right\|_{L^{2}}+\left\|c_{0}\right\|_{L^{2}}\right)\left\|w_{0}\right\|_{V}-\left(a_{1}+c_{1}\right) T\left\|w_{0}\right\|_{V}^{2}-\left(a_{1}+c_{1}\right)\left\|w_{0}\right\|_{V} \sqrt{T}\|v\|_{\mathcal{V}}
\end{aligned}
$$

for all $v \in \mathcal{V}$. Finally, by the inequality $(a+b)^{2} \geq \frac{1}{2} a^{2}-b^{2}$ for all $a, b \in \mathbb{R}$, we conclude

$$
\begin{aligned}
& \langle\mathcal{F} v, v\rangle_{\mathcal{V}^{*} \times \mathcal{V}} \geq \frac{1}{2}\left(m_{A}-m_{\psi}\right)\|v\|_{\mathcal{V}}^{2}-\left(m_{A}-m_{\psi}\right)\left\|w_{0}\right\|_{\mathcal{V}}^{2}-\left(\left\|a_{0}\right\|_{L^{2}}+\left\|c_{0}\right\|_{L^{2}}\right)\|v\|_{\mathcal{V}} \\
& \quad-\left(\left\|a_{0}\right\|_{L^{2}}+\left\|c_{0}\right\|_{L^{2}}\right)(1+\sqrt{T})\left\|w_{0}\right\|_{\mathcal{V}}-\left(a_{1}+c_{1}\right) T\left\|w_{0}\right\|_{V}^{2}-\left(a_{1}+c_{1}\right)\left\|w_{0}\right\|_{V} \sqrt{T}\|v\|_{\mathcal{V}} .
\end{aligned}
$$

From the smallness hypothesis $\left(H_{1}\right)$, we infer that $\mathcal{F}$ is a coercive operator. The rest of the proof of this theorem is analogous to [8, Theorem 6], and therefore, it is omitted here.

Observe that existence and uniqueness result of Theorem 3 was proved earlier in [8] under the more restrictive smallness assumption $m_{A}>\max \left\{m_{\psi}, 2 \sqrt{2} c_{1}\right\}$.

Note that condition $H(\psi)$ (iv) has an equivalent formulation in terms of the generalized directional derivative of $\psi(t, \cdot)$, that is, $\partial \psi(t, \cdot)$ is relaxed monotone for a.e. $t \in(0, T)$ with constant $m_{\psi} \geq 0$ if and only if

$$
\psi^{0}\left(t, v_{1} ; v_{2}-v_{1}\right)+\psi^{0}\left(t, v_{2} ; v_{1}-v_{2}\right) \leq m_{\psi}\left\|v_{1}-v_{2}\right\|_{V}^{2}
$$

for all $v_{i} \in V, i=1,2$, a.e. $t \in(0, T)$. For details and examples, see, e.g., [7, Remark 3.1], and [19,20,32].

We pass now to the evolution inclusion with history-dependent operators which is the main object of our study in this paper.

Problem 4. Find $w \in \mathcal{W}$ such that

$$
\left\{\begin{array}{l}
w^{\prime}(t)+A(t, w(t))+\left(R_{1} w\right)(t)+M^{*} \partial J(t,(S w)(t), M w(t)) \\
\quad+N^{*} \partial \varphi(t,(R w)(t), N w(t)) \ni f(t) \text { a.e. } t \in(0, T) \\
w(0)=w_{0}
\end{array}\right.
$$

In this problem, $\partial J$ denotes the generalized gradient of a locally Lipschitz function $J(t, z, \cdot)$ and $\partial \varphi$ is the convex subdifferential of a convex and lower semicontinuous function $\varphi(t, y, \cdot)$. Despite two subdifferential terms generated by convex and (in general) nonconvex functions, the inclusion involves three nonlinear operators $R, R_{1}$ and $S$ called history-dependent ones.

We introduce the following hypotheses on the data of Problem 4. Let $X, Y, Z$ and $U$ be Banach spaces.

$H(J): J:(0, T) \times Z \times X \rightarrow \mathbb{R}$ is such that

(i) $J(\cdot, z, v)$ is measurable on $(0, T)$ for all $z \in Z, v \in X$.

(ii) $J(t, \cdot, v)$ is continuous on $Z$ for all $v \in X$, a.e. $t \in(0, T)$.

(iii) $J(t, z, \cdot)$ is locally Lipschitz on $X$ for all $z \in Z$, a.e. $t \in(0, T)$. 
(iv) $\|\partial J(t, z, v)\|_{X^{*}} \leq c_{0 J}(t)+c_{1 J}\|z\|_{Z}+c_{2 J}\|v\|_{X}$ for all $z \in Z, v \in X$, a.e. $t \in(0, T)$ with $c_{0 J} \in L^{2}(0, T)$, $c_{0 J}, c_{1 J}, c_{2 J} \geq 0$.

(v) $\partial J$ is relaxed monotone in the following sense

$$
\begin{aligned}
& \left\langle\partial J\left(t, z_{1}, v_{1}\right)-\partial J\left(t, z_{2}, v_{2}\right), v_{1}-v_{2}\right\rangle_{X^{*} \times X} \\
& \quad \geq-m_{J}\left\|v_{1}-v_{2}\right\|_{X}^{2}-\bar{m}_{J}\left\|z_{1}-z_{2}\right\|_{Z}\left\|v_{1}-v_{2}\right\|_{X}
\end{aligned}
$$

for all $z_{i} \in Z, v_{i} \in X, i=1,2$, a.e. $t \in(0, T)$ with $m_{J} \geq 0, \bar{m}_{J} \geq 0$.

$H(\varphi): \varphi:(0, T) \times Y \times U \rightarrow \mathbb{R}$ is such that

(i) $\varphi(\cdot, y, u)$ is measurable on $(0, T)$ for all $y \in Y, u \in U$

(ii) $\varphi(t, \cdot, u)$ is continuous on $Y$ for all $u \in U$, a.e. $t \in(0, T)$.

(iii) $\varphi(t, y, \cdot)$ is convex and l.s.c. on $U$ for all $y \in Y$, a.e. $t \in(0, T)$.

(iv) $\|\partial \varphi(t, y, u)\|_{U^{*}} \leq c_{0 \varphi}(t)+c_{1 \varphi}\|y\|_{Y}+c_{2 \varphi}\|u\|_{U}$ for all $y \in Y, u \in U$, a.e. $t \in(0, T)$ with $c_{0 \varphi} \in L^{2}(0, T)$, $c_{0 \varphi}, c_{1 \varphi}, c_{2 \varphi} \geq 0$.

(v) $\varphi\left(t, y_{1}, u_{2}\right)-\varphi\left(t, y_{1}, u_{1}\right)+\varphi\left(t, y_{2}, u_{1}\right)-\varphi\left(t, y_{2}, u_{2}\right) \leq \beta_{\varphi}\left\|y_{1}-y_{2}\right\|_{Y}\left\|u_{1}-u_{2}\right\|_{U}$ for all $y_{i} \in Y, u_{i} \in U$, $i=1,2$, a.e. $t \in(0, T)$ with $\beta_{\varphi} \geq 0$.

$H(M, N): M \in \mathcal{L}(V, X), N \in \mathcal{L}(V, U)$.

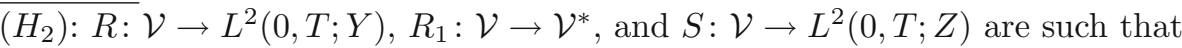

(i) $\left\|\left(R v_{1}\right)(t)-\left(R v_{2}\right)(t)\right\|_{Y} \leq c_{R} \int_{0}^{t}\left\|v_{1}(s)-v_{2}(s)\right\|_{V} \mathrm{~d} s$ for all $v_{1}, v_{2} \in \mathcal{V}$, a.e. $t \in(0, T)$ with $c_{R}>0$.

(ii) $\left\|\left(R_{1} v_{1}\right)(t)-\left(R_{1} v_{2}\right)(t)\right\|_{V^{*}} \leq c_{R_{1}} \int_{0}^{t}\left\|v_{1}(s)-v_{2}(s)\right\|_{V} \mathrm{~d} s$ for all $v_{1}, v_{2} \in \mathcal{V}$, a.e. $t \in(0, T)$ with $c_{R_{1}}>0$.

(iii) $\left\|\left(S v_{1}\right)(t)-\left(S v_{2}\right)(t)\right\|_{Z} \leq c_{S} \int_{0}^{t}\left\|v_{1}(s)-v_{2}(s)\right\|_{V} \mathrm{~d} s$ for all $v_{1}, v_{2} \in \mathcal{V}$, a.e. $t \in(0, T)$ with $c_{S}>0$. $\underline{\left(H_{3}\right)}: m_{A}>m_{J}\|M\|^{2}$.

Theorem 5. Under hypotheses $H(A), H(J), H(\varphi), H(M, N),\left(H_{0}\right),\left(H_{2}\right)$ and $\left(H_{3}\right)$, Problem 4 has a unique solution.

Proof. The proof is based on Theorem 3, uses some ideas from [8, Theorem 9] and consists of three steps.

Step 1 Let us fix $\xi \in L^{2}\left(0, T ; V^{*}\right), \eta \in L^{2}(0, T ; Y)$ and $\zeta \in L^{2}(0, T ; Z)$ and define a functional $\psi_{\xi \eta \zeta}:(0, T) \times V \rightarrow \mathbb{R}$ by

$$
\psi_{\xi \eta \zeta}(t, v)=\langle\xi(t), v\rangle_{V^{*} \times V}+J(t, \zeta(t), M v)+\varphi(t, \eta(t), N v)
$$

for all $v \in V$, a.e. $t \in(0, T)$.

We will verify that $\psi_{\xi \eta \zeta}$ satisfies hypothesis $H(\psi)$. First, it is clear by assumptions $H(J)(\mathrm{i})$, (ii) and $H(\varphi)(\mathrm{i})$, (ii) that both $J(\cdot, \cdot, v)$ and $\varphi(\cdot, \cdot, u)$ are Carathéodory functions for all $v \in V, u \in U$. Making compositions with measurable functions $(0, T) \ni t \mapsto \zeta(t) \in Y$ and $(0, T) \ni t \mapsto \eta(t) \in Z$, it is obvious that the functional $\psi_{\xi \eta \zeta}(\cdot, v)$ is measurable on $(0, T)$ for all $v \in V$. This implies $H(\psi)(\mathrm{i})$. Next, we observe that $H(\psi)$ (ii) is satisfied. Indeed, using [3, Proposition 5.2.10] and $H(\varphi)($ iii), we infer that $\varphi(t, y, \cdot)$ is locally Lipschitz on $U$ for all $y \in Y$, a.e. $t \in(0, T)$. Hence, taking into account $H(J)$ (iii) combined with $H(M, N)$, we obtain that the functional $\psi_{\xi \eta \zeta}(t, \cdot)$ is locally Lipschitz on $V$ for a.e. $t \in(0, T)$, i.e., $H(\psi)($ ii) holds.

Subsequently, since $J(t, z, M \cdot)$ and $\varphi(t, y, N \cdot)$ are locally Lipschitz for all $z \in Z$ and $y \in Y$, a.e. $t \in(0, T)$, we apply the chain rule in [3, Proposition 5.6.23] to get

$$
\partial \psi_{\xi \eta \zeta}(t, v) \subset \xi(t)+M^{*} \partial J(t, \zeta(t), M v)+N^{*} \partial \varphi(t, \eta(t), N v)
$$


for all $v \in V$ and a.e. $t \in(0, T)$. Exploiting (3.3), we easily have

$$
\begin{aligned}
\left\|\partial \psi_{\xi \eta \zeta}(t, v)\right\|_{V^{*}} \leq & \|\xi(t)\|_{V^{*}}+\left\|M^{*} \partial J(t, \zeta(t), M v)\right\|_{V^{*}}+\left\|N^{*} \partial \varphi(t, \eta(t), N v)\right\|_{V^{*}} \\
\leq & \|\xi(t)\|_{V^{*}}+c_{0 J}(t)+c_{1 J}\|M\|\|\zeta(t)\|_{Z}+c_{2 J}\|M\|^{2}\|v\|_{V} \\
& +c_{0 \varphi}(t)+c_{1 \varphi}\|N\|\|\eta(t)\|_{Y}+c_{2 \varphi}\|N\|^{2}\|v\|_{V} \\
\leq & c_{0}(t)+c_{1}\|v\|_{V}
\end{aligned}
$$

for all $v \in V$, a.e. $t \in(0, T)$, where $c_{0} \in L^{2}(0, T), c_{0} \geq 0$ and

$$
c_{1}=\max \left\{c_{2 J}\|M\|^{2}, c_{2 \varphi}\|N\|^{2}\right\} \geq 0 .
$$

Condition $H(\psi)($ iii) follows.

Finally, we will check the relaxed monotonicity condition $H(\psi)(\mathrm{iv})$. We use $H(J)(\mathrm{v})$ and the monotonicity of $\partial \varphi(t, y, \cdot)$ for all $y \in Y$, a.e. $t \in(0, T)$, see [3, Theorem 6.3.19], which entail

$$
\begin{aligned}
\left\langle\partial \psi_{\xi \eta \zeta}\left(t, v_{1}\right)-\partial \psi_{\xi \eta \zeta}\left(t, v_{2}\right), v_{1}-v_{2}\right\rangle_{V^{*} \times V} \\
=\left\langle\partial J\left(t, \zeta(t), M v_{1}\right)-\partial J\left(t, \zeta(t), M v_{2}\right), M v_{1}-M v_{2}\right\rangle_{X^{*} \times X} \\
\quad+\left\langle\partial \varphi\left(t, \eta(t), N v_{1}\right)-\partial \varphi\left(t, \eta(t), N v_{2}\right), N v_{1}-N v_{2}\right\rangle_{U^{*} \times U} \\
\geq-m_{J}\left\|M\left(v_{1}-v_{2}\right)\right\|_{X}^{2} \geq-m_{J}\|M\|^{2}\left\|v_{1}-v_{2}\right\|_{V}^{2}
\end{aligned}
$$

for all $v_{1}, v_{2} \in V$, a.e. $t \in(0, T)$. We deduce that condition $H(\psi)($ iv $)$ is satisfied with $m_{\psi}=m_{J}\|M\|^{2}$. This completes the proof of $H(\psi)$.

Since $m_{\psi}=m_{J}\|M\|^{2}$, hypothesis $\left(H_{3}\right)$ implies condition $\left(H_{1}\right)$. Therefore, by applying Theorem 3 , we obtain that there exists a unique element $w_{\xi \eta \zeta} \in \mathcal{W}$ which solves the inclusion in Problem 2 with $\psi$ replaced by $\psi_{\xi \eta \zeta}$. Moreover, by (3.3), it is clear that $w_{\xi \eta \zeta} \in \mathcal{W}$ is also a solution to the following problem: find $w \in \mathcal{W}$ such that

$$
\left\{\begin{array}{l}
w^{\prime}(t)+A(t, w(t))+\xi(t)+M^{*} \partial J(t, \zeta(t), M w(t)) \\
\quad+N^{*} \partial \varphi(t, \eta(t), N w(t)) \ni f(t) \text { a.e. } t \in(0, T), \\
w(0)=w_{0} .
\end{array}\right.
$$

Step 2 We claim that a solution to the problem (3.4) is unique. Let $w_{1}, w_{2} \in \mathcal{W}$ be solutions to the problem (3.4). For simplicity, we skip the subscripts $\xi, \eta$ and $\zeta$ in this part of the proof. Take $w_{2}(t)$ as the test function in the inclusion in (3.4) satisfied by $w_{1}$, take $w_{1}(t)$ as the test function in the inclusions (3.4) for $w_{2}$, and add the two resulting expressions. We have

$$
\begin{aligned}
& \left\langle w_{1}^{\prime}(t)-w_{2}^{\prime}(t), w_{1}(t)-w_{2}(t)\right\rangle_{V^{*} \times V}+\left\langle A\left(t, w_{1}(t)\right)-A\left(t, w_{2}(t)\right), w_{1}(t)-w_{2}(t)\right\rangle_{V^{*} \times V} \\
& \quad+\left\langle\partial J\left(t, \zeta(t), M w_{1}(t)\right)-\partial J\left(t, \zeta(t), M w_{2}(t)\right), M w_{1}(t)-M w_{2}(t)\right\rangle_{X^{*} \times X} \\
& \quad+\left\langle\partial \varphi\left(t, \eta(t), N w_{1}(t)\right)-\partial \varphi\left(t, \eta(t), N w_{2}(t)\right), N w_{1}(t)-N w_{2}(t)\right\rangle_{U^{*} \times U}=0
\end{aligned}
$$

for a.e. $t \in(0, T)$. Next, we integrate the above inequality on $(0, t)$, for all $t \in[0, T]$ and then use the integration by parts, $H(A)(\mathrm{iv}), H(J)(\mathrm{v})$, the monotonicity of the convex subdifferential and condition $w_{1}(0)-w_{2}(0)=0$ to deduce

$$
\frac{1}{2}\left\|w_{1}(t)-w_{2}(t)\right\|_{H}^{2}+m_{A} \int_{0}^{t}\left\|w_{1}(s)-w_{2}(s)\right\|_{V}^{2} \mathrm{~d} s-m_{J} \int_{0}^{t}\left\|w_{1}(s)-w_{2}(s)\right\|_{V}^{2} \mathrm{~d} s \leq 0
$$

for all $t \in[0, T]$. By the smallness condition $\left(H_{3}\right)$, we conclude $w_{1}=w_{2}$. The solution to problem (3.4) is unique.

Step 3 Consider operator $\Lambda: L^{2}\left(0, T ; V^{*} \times Y \times Z\right) \rightarrow L^{2}\left(0, T ; V^{*} \times Y \times Z\right)$ by

$$
\Lambda(\xi, \eta, \zeta)=\left(R_{1} w_{\xi \eta \zeta}, R w_{\xi \eta \zeta}, S w_{\xi \eta \zeta}\right) \quad \text { for all }(\xi, \eta, \zeta) \in L^{2}\left(0, T ; V^{*} \times Y \times Z\right) \text {, }
$$


where $w_{\xi \eta \zeta} \in \mathcal{W}$ denotes the unique solution to the problem (3.4) corresponding to $(\xi, \eta, \zeta)$. By an argument similar to the one used in [8, Theorem 9 and Lemma 3], we deduce that there exists a unique fixed point $\left(\xi^{*}, \eta^{*}, \zeta^{*}\right)$ of $\Lambda$, i.e.,

$$
\left(\xi^{*}, \eta^{*}, \zeta^{*}\right) \in L^{2}\left(0, T ; V^{*} \times Y \times Z\right) \text { and } \Lambda\left(\xi^{*}, \eta^{*}, \zeta^{*}\right)=\left(\xi^{*}, \eta^{*}, \zeta^{*}\right) .
$$

Let $w_{\xi^{*}} \eta^{*} \zeta^{*} \in \mathcal{W}$ be the unique solution to the problem (3.4) corresponding to $\left(\xi^{*}, \eta^{*}, \zeta^{*}\right)$. By definition of operator $\Lambda$, we have

$$
\xi^{*}=R_{1}\left(w_{\xi^{*}} \eta^{*} \zeta^{*}\right), \quad \eta^{*}=R\left(w_{\xi^{*}} \eta^{*} \zeta^{*}\right) \quad \text { and } \quad \zeta^{*}=S\left(w_{\xi^{*}} \eta^{*} \zeta^{*}\right) .
$$

Finally, we use these equalities in (3.4), and conclude $w_{\xi^{*}} \eta^{*} \zeta^{*}$ is the unique solution of Problem 4. This completes the proof of the theorem.

\section{A continuous dependence result}

In this section, we provide a new continuous dependence result for Problem 4. We study the continuity in the weak topologies of the map which to the right-hand side and initial condition in Problem 4 assigns its unique solution.

We will prove first the following a priori estimate on a solution.

Proposition 6. Under hypotheses of Theorem 5, if $w \in \mathcal{W}$ is a solution to Problem 4, then there exists a constant $C>0$ such that

$$
\begin{aligned}
& \|w\|_{C(0, T ; H)}+\|w\|_{\mathcal{W}} \leq C\left(1+\left\|w_{0}\right\|_{V}+\|f\|_{\mathcal{V}^{*}}+\|R 0\|_{L^{2}(0, T ; Y)}\right. \\
& \left.\quad+\left\|R_{1} 0\right\|_{L^{2}\left(0, T ; V^{*}\right)}+\|S 0\|_{L^{2}(0, T ; Z)}\right) .
\end{aligned}
$$

Proof. Let us denote by $w \in \mathcal{W}$ a solution to Problem 4 . This means that there are $\xi \in L^{2}\left(0, T ; X^{*}\right)$ and $\eta \in L^{2}\left(0, T ; U^{*}\right)$ such that

$$
\begin{aligned}
& w^{\prime}(t)+A(t, w(t))+\left(R_{1} w\right)(t)+M^{*} \xi(t)+N^{*} \eta(t)=f(t) \text { a.e. } t \in(0, T), \\
& \xi(t) \in \partial J(t,(S w)(t), M w(t)) \text { a.e. } t \in(0, T), \\
& \eta(t) \in \partial \varphi(t,(R w)(t), N w(t)) \text { a.e. } t \in(0, T), \\
& w(0)=w_{0} .
\end{aligned}
$$

We take the duality with $w(t)$ in $(4.1)$ to get

$$
\begin{aligned}
& \left\langle w^{\prime}(t)+A(t, w(t))+\left(R_{1} w\right)(t), w(t)\right\rangle_{V^{*} \times V}+\langle\xi(t), M w(t)\rangle_{X^{*} \times X} \\
& \quad+\langle\eta(t), N w(t)\rangle_{Y^{*} \times Y}=\langle f(t), w(t)\rangle_{V^{*} \times V} .
\end{aligned}
$$

In the estimates below, we use several times the Hölder inequality, Young's inequality $a b \leq \frac{\varepsilon^{2}}{2} a^{2}+\frac{1}{2 \varepsilon^{2}} b^{2}$ with $\varepsilon>0$, and elementary inequality $(a+b)^{2} \leq 2\left(a^{2}+b^{2}\right)$ for all $a, b \in \mathbb{R}$. Let $t \in[0, T]$. From hypothesis $\left(H_{2}\right)$, we have

$$
\begin{aligned}
& \|(R w)(t)\|_{Y}^{2} \leq 2 c_{R}^{2} t \int_{0}^{t}\|w(s)\|_{V}^{2} \mathrm{~d} s+2\|(R 0)(t)\|_{Y}^{2}, \\
& \left\|\left(R_{1} w\right)(t)\right\|_{V^{*}}^{2} \leq 2 c_{R_{1}}^{2} t \int_{0}^{t}\|w(s)\|_{V}^{2} \mathrm{~d} s+2\left\|\left(R_{1} 0\right)(t)\right\|_{V^{*}}^{2}, \\
& \|(S w)(t)\|_{Z}^{2} \leq 2 c_{S}^{2} t \int_{0}^{t}\|w(s)\|_{V}^{2} \mathrm{~d} s+2\|(S 0)(t)\|_{Z}^{2} .
\end{aligned}
$$


By assumption $H(\varphi)($ iv) and (4.6), we obtain

$$
\begin{aligned}
& \left|\langle\partial \varphi(t,(R w)(t), 0), N w(t)\rangle_{Y^{*} \times Y}\right| \leq\left(c_{0 \varphi}(t)+c_{1 \varphi}\|(R w)(t)\|_{Y}\right)\|N\|\|w(t)\|_{V} \\
& \quad \leq \frac{\varepsilon^{2}}{2}\|N\|^{2}\|w(t)\|_{V}^{2}+\frac{1}{\varepsilon^{2}}\left(c_{0 \varphi}^{2}(t)+c_{1 \varphi}^{2}\|(R w)(t)\|_{Y}^{2}\right) \\
& \quad \leq \frac{\varepsilon^{2}}{2}\|N\|^{2}\|w(t)\|_{V}^{2}+\frac{c_{0 \varphi}^{2}(t)}{\varepsilon^{2}}+\frac{2 c_{1 \varphi}^{2} c_{R}^{2} T}{\varepsilon^{2}} \int_{0}^{t}\|w(s)\|_{V}^{2} \mathrm{~d} s+\frac{2 c_{1 \varphi}^{2}}{\varepsilon^{2}}\|(R 0)(t)\|_{Y}^{2} .
\end{aligned}
$$

The latter combined with (4.3) and the monotonicity of the convex subdifferential implies

$$
\begin{aligned}
& \langle\eta(t), N w(t)\rangle_{Y^{*} \times Y}=\langle\partial \varphi(t,(R w)(t), N w(t)), N w(t)\rangle_{Y^{*} \times Y} \\
& \quad \geq\langle\partial \varphi(t,(R w)(t), 0), N w(t)\rangle_{Y^{*} \times Y} \\
& \quad \geq-\frac{\varepsilon^{2}}{2}\|N\|^{2}\|w(t)\|_{V}^{2}-\frac{c_{0 \varphi}^{2}(t)}{\varepsilon^{2}}-\frac{2 c_{1 \varphi}^{2} c_{R}^{2} T}{\varepsilon^{2}} \int_{0}^{t}\|w(s)\|_{V}^{2} \mathrm{~d} s-\frac{2 c_{1 \varphi}^{2}}{\varepsilon^{2}}\|(R 0)(t)\|_{Y}^{2} .
\end{aligned}
$$

Similarly, by hypotheses $H(J)($ iv) and (v), (4.2), and (4.8), we get

$$
\begin{gathered}
\langle\xi(t), M w(t)\rangle_{X^{*} \times X}=\langle\partial J(t,(S w)(t), M w(t)), M w(t)\rangle_{X^{*} \times X} \\
\geq\langle\partial J(t,(S w)(t), 0), M w(t)\rangle_{Y^{*} \times Y}-m_{J}\|M\|^{2}\|w(t)\|_{V}^{2} \\
\geq-m_{J}\|M\|^{2}\|w(t)\|_{V}^{2}-\frac{\varepsilon^{2}}{2}\|M\|^{2}\|w(t)\|_{V}^{2}-\frac{c_{0 J}^{2}(t)}{\varepsilon^{2}} \\
\quad-\frac{2 c_{1 J}^{2} c_{S}^{2} T}{\varepsilon^{2}} \int_{0}^{t}\|w(s)\|_{V}^{2} \mathrm{~d} s-\frac{2 c_{1 J}^{2}}{\varepsilon^{2}}\|(S 0)(t)\|_{Z}^{2} .
\end{gathered}
$$

Next, exploiting (4.7), we have

$$
\begin{aligned}
& \left.\int_{0}^{t}\left\langle\left(R_{1} w\right)(s)\right), w(s)\right\rangle_{V^{*} \times V} \mathrm{~d} s \leq \frac{\varepsilon^{2}}{2} \int_{0}^{t}\|w(s)\|_{V}^{2} \mathrm{~d} s \\
& \quad+\frac{c_{R_{1}}^{2} t}{\varepsilon^{2}} \int_{0}^{t}\left(\int_{0}^{s}\|w(\tau)\|_{V}^{2} \mathrm{~d} \tau\right) \mathrm{d} s+\frac{1}{\varepsilon^{2}} \int_{0}^{t}\left\|\left(R_{1} 0\right)(s)\right\|_{V^{*}}^{2} \mathrm{~d} s .
\end{aligned}
$$

On the other hand, a simple calculation gives

$$
\begin{gathered}
\left.\int_{0}^{t}\langle f(s)), w(s)\right\rangle_{V^{*} \times V} \mathrm{~d} s \leq \frac{\varepsilon^{2}}{2} \int_{0}^{t}\|w(s)\|_{V}^{2} \mathrm{~d} s+\frac{1}{2 \varepsilon^{2}} \int_{0}^{t}\|f(s)\|_{V^{*}}^{2} \mathrm{~d} s, \\
\int_{0}^{t} a_{0}(s)\|w(s)\|_{V} \mathrm{~d} s \leq \frac{\varepsilon^{2}}{2}\|w\|_{L^{2}(0, t ; V)}^{2}+\frac{1}{2 \varepsilon^{2}}\left\|a_{0}\right\|_{L^{2}(0, T)}^{2} .
\end{gathered}
$$

Subsequently, we integrate $(4.5)$ on $(0, t)$ for all $t \in[0, T]$, use integration by parts formula in [4, Proposition 8.4.14], $H(A)($ iii) and (iv), and inequalities (4.9)-(4.13), to deduce

$$
\begin{aligned}
\frac{1}{2} \| w(t) & \left\|_{H}^{2}+\left(m_{A}-m_{J}\|M\|^{2}-\frac{3 \varepsilon^{2}}{2}-\frac{\varepsilon^{2}}{2}\left(\|N\|^{2}+\|M\|^{2}\right)\right) \int_{0}^{t}\right\| w(s) \|_{V}^{2} \mathrm{~d} s \\
\leq & \frac{1}{2}\left\|w_{0}\right\|_{H}^{2}+\frac{1}{2 \varepsilon^{2}}\left\|a_{0}\right\|_{L^{2}(0, T)}^{2}+\frac{1}{\varepsilon^{2}}\left(\left\|c_{0 \varphi}\right\|_{L^{2}(0, T)}^{2}+\left\|c_{0 J}\right\|_{L^{2}(0, T)}^{2}\right) \\
& +\frac{1}{\varepsilon^{2}}\left(2 c_{1 \varphi}^{2} c_{R}^{2} T+2 c_{1 J}^{2} c_{S}^{2} T+c_{R_{1}}^{2} T\right) \int_{0}^{t}\left(\int_{0}^{s}\|w(\tau)\|_{V}^{2} \mathrm{~d} \tau\right) \mathrm{d} s+\frac{1}{2 \varepsilon^{2}} \int_{0}^{t}\|f(s)\|_{V^{*}}^{2} \mathrm{~d} s \\
& +\frac{2 c_{1 \varphi}^{2}}{\varepsilon^{2}} \int_{0}^{t}\|(R 0)(s)\|_{Y}^{2} \mathrm{~d} s+\frac{1}{\varepsilon^{2}} \int_{0}^{t}\left\|\left(R_{1} 0\right)(s)\right\|_{V^{*}}^{2} \mathrm{~d} s+\frac{2 c_{1 J}^{2}}{\varepsilon^{2}} \int_{0}^{t}\|(S 0)(s)\|_{Z}^{2} \mathrm{~d} s .
\end{aligned}
$$

Now, by $\left(H_{3}\right)$, we choose $\varepsilon>0$ such that

$$
m_{A}-m_{J}\|M\|^{2}-\frac{3 \varepsilon^{2}}{2}-\frac{\varepsilon^{2}}{2}\left(\|N\|^{2}+\|M\|^{2}\right)>0 .
$$


Hence, for some positive constants $d_{i}, i=1, \ldots, 5$, we have

$$
\begin{gathered}
d_{1} \int_{0}^{t}\|w(s)\|_{V}^{2} \mathrm{~d} s \leq \frac{1}{2}\left\|w_{0}\right\|_{H}^{2}+d_{2}\|f\|_{L^{2}\left(0, t ; V^{*}\right)}^{2}+d_{3} \int_{0}^{t}\left(\int_{0}^{s}\|w(\tau)\|_{V}^{2} \mathrm{~d} \tau\right) \mathrm{d} s \\
\quad+d_{4} \int_{0}^{t}\left(\|(R 0)(s)\|_{Y}^{2}+\left\|\left(R_{1} 0\right)(s)\right\|_{V^{*}}^{2}+\|(S 0)(s)\|_{Z}^{2}\right) \mathrm{d} s+d_{5}
\end{gathered}
$$

for all $t \in[0, T]$. Applying the Gronwall lemma, see, e.g., [19, Lemma 2.7], we deduce the desired estimate on the term $\|w\|_{\mathcal{V}}$. By (4.1), we obtain the bound on $\left\|w^{\prime}\right\|_{\mathcal{V}^{*}}$, and finally also on the norm of the solution $w$ in $C(0, T ; H)$. This proves the estimate in the statement of the proposition and completes the proof.

To provide a result on the continuous dependence, we need stronger versions of hypotheses introduced in the previous section. In particular, operator $A$ will be assumed to be time independent and the weaklyweakly continuous which obviously implies the demicontinuity in $H(A)$ (ii). All hypotheses introduced below are clearly satisfied in applications in Sects. 5 and 6 .

$H(A)_{1}: A: V \rightarrow V^{*}$ is such that

(i) $A$ is weakly-weakly continuous.

(ii) $\|A v\|_{V^{*}} \leq a_{0}+a_{1}\|v\|_{V}$ for all $v \in V$ with $a_{0}, a_{1} \geq 0$.

(iii) $A$ is strongly monotone with constant $m_{A}>0$, i.e.,

$$
\left\langle A v_{1}-A v_{2}, v_{1}-v_{2}\right\rangle_{V^{*} \times V} \geq m_{A}\left\|v_{1}-v_{2}\right\|_{V}^{2}
$$

for all $v_{1}, v_{2} \in V$.

$\underline{H(J)_{1}}: J:(0, T) \times Z \times X \rightarrow \mathbb{R}$ is such that $H(J)(\mathrm{i})-(\mathrm{v})$ hold and

(vi) $J^{0}(t, \cdot, \cdot ; w): Z \times X \rightarrow \mathbb{R}$ is upper semicontinuous on $Z \times X$ for all $w \in X$.

$H(M, N)_{1}: M$ and $N$ satisfy $H(M, N)$, and their Nemytskii operators

$$
\mathcal{M}: \mathcal{W} \subset \mathcal{V} \rightarrow L^{2}(0, T ; X) \text { and } \mathcal{N}: \mathcal{W} \subset \mathcal{V} \rightarrow L^{2}(0, T ; U)
$$

are compact.

$\left(H_{4}\right): R, R_{1}$ and $S$ satisfy $\left(H_{2}\right)$, and

(i) $R: \mathcal{W} \subset \mathcal{V} \rightarrow L^{2}(0, T ; Y)$ and $S: \mathcal{W} \subset \mathcal{V} \rightarrow L^{2}(0, T ; Z)$ are compact.

(ii) $R_{1}: \mathcal{V} \rightarrow \mathcal{V}^{*}$ is weakly-weakly continuous.

(iii) $\left(R 0, R_{1} 0, S 0\right)$ remains in a bounded subset of $L^{2}\left(0, T ; Y \times V^{*} \times Z\right)$.

Theorem 7. Under hypotheses $H(A)_{1}, H(J)_{1}, H(\varphi), H(M, N)_{1},\left(H_{0}\right),\left(H_{3}\right)$, and $\left(H_{4}\right)$, if $\left\{f_{n}\right\} \subset \mathcal{V}^{*}$, $f_{n} \rightarrow f$ weakly in $\mathcal{V}^{*},\left\{w_{0}^{n}\right\} \subset V, w_{0}^{n} \rightarrow w_{0}$ weakly in $V$, and $\left\{w_{n}\right\} \subset \mathcal{W}, w \in \mathcal{W}$ are the unique solutions to Problem 4 corresponding to $\left\{\left(f_{n}, w_{0}^{n}\right)\right\}$ and $\left(f, w_{0}\right)$, respectively, then $w_{n} \rightarrow w$ weakly in $\mathcal{W}$, as $n \rightarrow \infty$.

Proof. The existence and uniqueness of solution is a consequence of Theorem 5 . We prove the continuous dependence result. Let $\left\{f_{n}\right\} \subset \mathcal{V}^{*}, f_{n} \rightarrow f$ weakly in $\mathcal{V}^{*},\left\{w_{0}^{n}\right\} \subset V, w_{0}^{n} \rightarrow w_{0}$ weakly in $V$, and $\left\{w_{n}\right\} \subset \mathcal{W}$ be the unique solution to Problem 4 corresponding to $\left\{\left(f_{n}, w_{0}^{n}\right)\right\}, n \in \mathbb{N}$. Then,

$$
\begin{aligned}
& w_{n}^{\prime}(t)+A w_{n}(t)+\left(R_{1} w_{n}\right)(t)+M^{*} \xi_{n}(t)+N^{*} \eta_{n}(t)=f_{n}(t) \text { a.e. } t \in(0, T), \\
& \xi_{n}(t) \in \partial J\left(t,\left(S w_{n}\right)(t), M w_{n}(t)\right) \text { a.e. } t \in(0, T), \\
& \eta_{n}(t) \in \partial \varphi\left(t,\left(R w_{n}\right)(t), N w_{n}(t)\right) \text { a.e. } t \in(0, T), \\
& w_{n}(0)=w_{0}^{n} .
\end{aligned}
$$

By Proposition 6 combined with $\left(H_{4}\right)$ (iii) and weak convergences of $\left\{f_{n}\right\}$ and $\left\{w_{0}^{n}\right\}$, the sequence $\left\{w_{n}\right\}$ is uniformly bounded in $\mathcal{W}$. By the reflexivity of $\mathcal{W}$, we can find a subsequence, denoted in the same way, such that $w_{n} \rightarrow w$ weakly in $\mathcal{W}$ with $w \in \mathcal{W}$, as $n \rightarrow \infty$. We will prove that $w$ is the unique solution in $\mathcal{W}$ to Problem 4 corresponding to $\left(f, w_{0}\right)$. 
Using an argument similar to [34, Lemma 13], from hypothesis $H(A)_{1}$, we known that

$$
\mathcal{A} w_{n} \rightarrow \mathcal{A} w \text { weakly in } \mathcal{V}^{*} \text {, as } n \rightarrow \infty \text {. }
$$

By assumption $\left(H_{4}\right)(\mathrm{ii})$, it follows

$$
R_{1} w_{n} \rightarrow R_{1} w \text { weakly in } \mathcal{V}^{*} \text {, as } n \rightarrow \infty \text {. }
$$

Further, we use $H(M, N)_{1}$ to get $\mathcal{M} w_{n} \rightarrow \mathcal{M} w$ in $L^{2}(0, T ; X)$ and $\mathcal{N} w_{n} \rightarrow \mathcal{N} w$ in $L^{2}(0, T ; U)$, which, for a next subsequence, entails

$$
M w_{n}(t) \rightarrow M w(t) \text { in } X \text {, and } N w_{n}(t) \rightarrow N w(t) \text { in } U \text {, for a.e. } t \in(0, T) .
$$

On the other hand, by $\left(H_{4}\right)(\mathrm{i})$, we have $R w_{n} \rightarrow R w$ in $L^{2}(0, T ; Y)$ and $S w_{n} \rightarrow S w$ in $L^{2}(0, T ; Z)$. Hence, again for a subsequence, it holds

$$
\left(R w_{n}\right)(t) \rightarrow(R w)(t) \text { in } Y, \text { and }\left(S w_{n}\right)(t) \rightarrow(S w)(t) \text { in } Z \text {, for a.e. } t \in(0, T) .
$$

Next, we prove the following claims.

Claim 1. If $J:(0, T) \times Z \times X \rightarrow \mathbb{R}$ satisfies $H(J)\left(\right.$ iii), (iv) and $H(J)_{1}($ vi), then the multivalued map

$$
Z \times X \ni(z, x) \mapsto \partial J(t, z, x) \subset X^{*}
$$

is upper semicontinuous from $Z \times X$ into $X^{*}$ endowed with the weak topology with nonempty, closed and convex values, for a.e. $t \in(0, T)$. Indeed, let us fix $t \in(0, T) \backslash N_{1}, m\left(N_{1}\right)=0$. Having in mind [3, Proposition 4.1.4], it is sufficient to show that for any weakly closed subset $D$ of $X^{*}$, the weak inverse image $(\partial J)^{-}(D)$ of $D$ under $\partial J(t, \cdot, \cdot)$ is closed in the norm topology, where $(\partial J)^{-}(D)$ is defined by

$$
(\partial J)^{-}(D)=\{(z, x) \in Z \times X \mid \partial J(t, z, x) \cap D \neq \emptyset\} .
$$

Let $\left\{\left(z_{n}, x_{n}\right)\right\} \subset(\partial J)^{-}(D)$ be such that $\left(z_{n}, x_{n}\right) \rightarrow(z, x)$ in $Z \times X$, as $n \rightarrow \infty$. Then, there is $\left\{\zeta_{n}\right\} \subset X^{*}$ such that $\zeta_{n} \in \partial J\left(t, z_{n}, x_{n}\right) \cap D$ for each $n \in \mathbb{N}$. Hypothesis $H(J)\left(\right.$ iv) implies that the sequence $\left\{\zeta_{n}\right\}$ is bounded in $X^{*}$. Hence, from the reflexivity of $X^{*}$, without any loss of generality, we may assume that $\zeta_{n} \rightarrow \zeta$ weakly in $X^{*}$. Since $D$ is weakly closed, we have $\zeta \in D$, and by condition $\zeta_{n} \in \partial J\left(t, z_{n}, x_{n}\right)$, we get

$$
\left\langle\zeta_{n}, w\right\rangle_{X^{*} \times X} \leq J^{0}\left(t, z_{n}, x_{n} ; w\right) \text { for all } w \in X
$$

Taking into account hypothesis $H(J)_{1}$ (vi) and passing to the limit, we have

$$
\langle\zeta, w\rangle_{X^{*} \times X}=\limsup _{n \rightarrow \infty}\left\langle\zeta_{n}, w\right\rangle_{X^{*} \times X} \leq \limsup _{n \rightarrow \infty} J^{0}\left(t, z_{n}, x_{n} ; w\right) \leq J^{0}(t, z, x ; w)
$$

for all $w \in X$. Thus, $\zeta \in \partial J(t, z, x)$, and finally, we get $\zeta \in \partial J(t, z, x) \cap D$, i.e., $(z, x) \in(\partial J)^{-}(D)$. Hence, $(\partial J)^{-}(D)$ is closed in $Z \times X$. The fact that the map $\partial J$ has nonempty, closed and convex values follows from, e.g., [19, Theorem 3.23(iv)]. This completes the proof of the claim.

Claim 2. If $\varphi:(0, T) \times Y \times U \rightarrow \mathbb{R}$ satisfies $H(\varphi)($ ii)-(iv), then the multivalued map

$$
Y \times U \ni(y, u) \mapsto \partial \varphi(t, y, u) \subset U^{*}
$$

is upper semicontinuous from $Y \times U$ into $U^{*}$ endowed with the weak topology with nonempty, closed and convex values for a.e. $t \in(0, T)$. This fact be proved similarly as Claim 1 . The map $\partial \varphi$ has nonempty, closed and convex values since $\varphi$ has finite values and we use [4, Proposition 6.3.10]. Let $t \in(0, T) \backslash N_{1}$ with $m\left(N_{1}\right)=0, E \subset U^{*}$ be weakly closed, and let

$$
(\partial \varphi)^{-}(E)=\{(y, u) \in Y \times U \mid \partial \varphi(t, y, u) \cap E \neq \emptyset\} .
$$

Let $\left\{\left(y_{n}, u_{n}\right)\right\} \subset(\partial \varphi)^{-}(E)$ and $\left(y_{n}, u_{n}\right) \rightarrow(y, u)$ in $Y \times U$, as $n \rightarrow \infty$. We can find $\left\{\rho_{n}\right\} \subset U^{*}$ such that $\rho_{n} \in \partial \varphi\left(t, y_{n}, u_{n}\right) \cap E$ for each $n \in \mathbb{N}$. It is clear from $H(\varphi)$ (iv) that the sequence $\left\{\rho_{n}\right\}$ is bounded in $U^{*}$, 
which by the reflexivity of $U^{*}$ entails, at least for a subsequence, $\rho_{n} \rightarrow \rho$ weakly in $U^{*}$. Obviously, $\rho \in E$ and

$$
\left\langle\rho_{n}, w\right\rangle_{U^{*} \times U} \leq \varphi\left(t, y_{n}, w\right)-\varphi\left(t, y_{n}, u_{n}\right) \text { for all } w \in U .
$$

Next, it follows from [3, Proposition 5.2.10] that $\varphi(t, y, \cdot)$ is locally Lipschitz. Hence, using $H(\varphi)($ ii) $-($ iv $)$, by [19, Lemma 3.43], we infer that $\varphi(t, \cdot, \cdot)$ is continuous on $Y \times U$. This allows to pass to the limit below

$$
\begin{aligned}
& \langle\rho, w-u\rangle_{U^{*} \times U}=\limsup _{n \rightarrow \infty}\left\langle\rho_{n}, w-u_{n}\right\rangle_{U^{*} \times U} \\
& \quad \leq \lim _{n \rightarrow \infty}\left(\varphi\left(t, y_{n}, w\right)-\varphi\left(y, y_{n}, u_{n}\right)\right)=\varphi(t, y, w)-\varphi(t, y, u)
\end{aligned}
$$

for all $w \in U$. This means that $\rho \in \partial \varphi(t, y, u) \cap E$ and finally $(y, u) \in(\partial \varphi)^{-}(E)$. Hence, the set $(\partial \varphi)^{-}(E)$ is closed in $Y \times U$, which by [3, Proposition 4.1.4] implies the desired upper semicontinuity of $\partial \varphi(t, \cdot, \cdot)$.

We now pass to the limits in (4.15) and (4.16). In both cases, we apply a convergence theorem of Aubin-Cellina [1], in a version provided in [16, Proposition 2] or in [27, Lemma 2.6]. Exploiting hypotheses $H(J)\left(\right.$ iv) and $H(\varphi)\left(\right.$ iv), it follows that sequences $\left\{\xi_{n}\right\}$ and $\left\{\eta_{n}\right\}$ are uniformly bounded in $L^{2}\left(0, T ; X^{*}\right)$ and $L^{2}\left(0, T ; U^{*}\right)$, respectively. Hence, again by passing to a subsequence if necessary, we may assume that

$$
\xi_{n} \rightarrow \xi \text { weakly in } L^{2}\left(0, T ; X^{*}\right) \text { and } \eta_{n} \rightarrow \eta \text { weakly in } L^{2}\left(0, T ; U^{*}\right)
$$

with $(\xi, \eta) \in L^{2}\left(0, T ; X^{*} \times U^{*}\right)$.

From convergences $(4.20),(4.21),(4.22)$ and properties of $\partial J$ of Claim 1, we apply the aforementioned convergence theorem to deduce

$$
\xi(t) \in \partial J(t,(S w)(t), M w(t)) \text { a.e. } t \in(0, T) .
$$

In a similar way, by $(4.20),(4.21),(4.22)$ and Claim 2 , we are able to apply the same convergence theorem to get

$$
\eta(t) \in \partial \varphi(t,(R w)(t), N w(t)) \text { a.e. } t \in(0, T) .
$$

On the other hand, it is immediate to see that

$$
\mathcal{M}^{*} \xi_{n} \rightarrow \mathcal{M}^{*} \xi \text { and } \mathcal{N}^{*} \eta_{n} \rightarrow \mathcal{N}^{*} \eta \text { weakly in } \mathcal{V}^{*}
$$

Since the map

$$
\mathcal{W} \ni w \mapsto w(0) \in H
$$

is linear and continuous, from convergence $w_{n} \rightarrow w$ weakly in $\mathcal{W}$, we have $w_{n}(0) \rightarrow w(0)$ weakly in $H$. Passing to the weak limit in $H$ in (4.17), we obtain $w(0)=w_{0}$. Applying convergences $w_{n}^{\prime} \rightarrow w^{\prime},(4.18)$, (4.19), (4.25), and $f_{n} \rightarrow f$ weakly in $\mathcal{V}^{*}$, we take the limit in $w_{n}^{\prime}+\mathcal{A} w_{n}+R_{1} w_{n}+\mathcal{M}^{*} \xi_{n}+\mathcal{N}^{*} \eta_{n}=f_{n}$ in $\mathcal{V}^{*}$. Hence,

$$
w^{\prime}+\mathcal{A} w+R_{1} w+\mathcal{M}^{*} \xi+\mathcal{N}^{*} \eta=f \text { in } \mathcal{V}^{*} .
$$

The latter combined with (4.23), (4.24) and $w(0)=w_{0}$ imply that that $w \in \mathcal{W}$ is a solution to Problem 4 corresponding to $\left(f, w_{0}\right)$. Since the solution is unique, we conclude that the whole sequence $\left\{w_{n}\right\}$ converges weakly in $\mathcal{W}$ to $w$. This completes the proof of the theorem.

\section{Application to a frictional contact problem}

In this section, we provide new continuous dependence results to a dynamic viscoelastic contact problem with friction which in its weak form leads to a history-dependent evolution inclusion analyzed in Sect. 4. Note that existence and uniqueness result for this problem has been obtained in [8] under a more restrictive smallness condition while the continuous dependence in weak topologies for this problem has not been studied before. 
We shortly recall the necessary notation and refer to $[8,19,30]$ for a detailed explanation and a discussion on mechanical interpretation. Let $\Omega \subset \mathbb{R}^{d}, d=2,3$, be a regular domain occupied in its reference configuration by a viscoelastic body. Its boundary $\Gamma$ consists of three disjoint measurable parts $\Gamma_{D}, \Gamma_{N}$ and $\Gamma_{C}$, such that $m\left(\Gamma_{D}\right)>0$. The body is clamped on $\Gamma_{D}$ (the displacement field vanishes there), the surface tractions act on $\Gamma_{N}$, and $\Gamma_{C}$ is a contact surface. All indices $i, j, k, l$ run between 1 and $d$ and, unless stated otherwise, the summation convention over repeated indices is applied. We use $\boldsymbol{u}=\left(u_{i}\right)$, $\boldsymbol{\sigma}=\left(\sigma_{i j}\right)$ and $\boldsymbol{\varepsilon}(\boldsymbol{u})=\left(\varepsilon_{i j}(\boldsymbol{u})\right)$ to denote the displacement vector, the stress tensor and linearized strain tensor, respectively. The latter is defined by

$$
\varepsilon_{i j}(\boldsymbol{u})=\frac{1}{2}\left(u_{i, j}+u_{j, i}\right)
$$

where $u_{i, j}=\partial u_{i} / \partial x_{j}$. For a vector field, we use the notation $v_{\nu}$ and $\boldsymbol{v}_{\tau}$ for the normal and tangential components of $\boldsymbol{v}$ on $\partial \Omega$ given by $v_{\nu}=\boldsymbol{v} \cdot \boldsymbol{\nu}$ and $\boldsymbol{v}_{\tau}=\boldsymbol{v}-v_{\nu} \boldsymbol{\nu}$, where $\boldsymbol{\nu}$ stands for the outward unit normal on the boundary. The normal and tangential components of the stress field $\sigma$ on the boundary are defined by $\sigma_{\nu}=(\boldsymbol{\sigma} \boldsymbol{\nu}) \cdot \boldsymbol{\nu}$ and $\boldsymbol{\sigma}_{\tau}=\boldsymbol{\sigma} \boldsymbol{\nu}-\sigma_{\nu} \boldsymbol{\nu}$, respectively. The symbol $\mathbb{S}^{d}$ stands for the space of symmetric matrices of order $d$, and the canonical inner products on $\mathbb{R}^{d}$ and $\mathbb{S}^{d}$ are given by $\boldsymbol{u} \cdot \boldsymbol{v}=u_{i} v_{i}$ for all $\boldsymbol{u}=\left(u_{i}\right), \boldsymbol{v}=\left(v_{i}\right) \in \mathbb{R}^{d}$, and $\boldsymbol{\sigma} \cdot \boldsymbol{\tau}=\sigma_{i j} \tau_{i j}$ for all $\boldsymbol{\sigma}=\left(\sigma_{i j}\right), \boldsymbol{\tau}=\left(\tau_{i j}\right) \in \mathbb{S}^{d}$, respectively.

We are interested in the evolution process of the mechanical state of the body, in the finite time interval. The classical formulation of the contact problem is stated as follows.

Problem 8. Find a displacement field $\boldsymbol{u}: \Omega \times(0, T) \rightarrow \mathbb{R}^{d}$ and a stress field $\boldsymbol{\sigma}: \Omega \times(0, T) \rightarrow \mathbb{S}^{d}$ such that for all $t \in(0, T)$,

$$
\begin{aligned}
\boldsymbol{\sigma}(t) & =\mathscr{A} \varepsilon\left(\boldsymbol{u}^{\prime}(t)\right)+\mathscr{B} \boldsymbol{\varepsilon}(\boldsymbol{u}(t))+\int_{0}^{t} \mathscr{C}(t-s) \varepsilon\left(\boldsymbol{u}^{\prime}(s)\right) \mathrm{d} s & & \text { in } \Omega, \\
\rho \boldsymbol{u}^{\prime \prime}(t) & =\operatorname{Div} \boldsymbol{\sigma}(t)+\boldsymbol{f}_{0}(t) & & \text { in } \Omega, \\
\boldsymbol{u}(t) & =\mathbf{0} & & \text { on } \Gamma_{D}, \\
\boldsymbol{\sigma}(t) \boldsymbol{\nu} & =\boldsymbol{f}_{2}(t) & & \text { on } \Gamma_{N}, \\
-\sigma_{\nu}(t) & \in k\left(u_{\nu}(t)\right) \partial j_{\nu}\left(u_{\nu}^{\prime}(t)\right) & & \text { on } \Gamma_{C}, \\
\left\|\boldsymbol{\sigma}_{\tau}(t)\right\| & \leq F_{b}\left(\int_{0}^{t}\left\|\boldsymbol{u}_{\tau}(s)\right\| \mathrm{d} s\right), & & \\
-\boldsymbol{\sigma}_{\tau} & =F_{b}\left(\int_{0}^{t}\left\|\boldsymbol{u}_{\tau}(s)\right\| \mathrm{d} s\right) \frac{\boldsymbol{u}_{\tau}^{\prime}(t)}{\left\|\boldsymbol{u}_{\tau}^{\prime}(t)\right\|} \text { if } \boldsymbol{u}_{\tau}^{\prime}(t) \neq \mathbf{0} & & \text { on } \Gamma_{C},
\end{aligned}
$$

and

$$
\boldsymbol{u}(0)=\boldsymbol{u}_{0}, \quad \boldsymbol{u}^{\prime}(0)=\boldsymbol{w}_{0} \quad \text { in } \Omega .
$$

Now, we recall the weak formulation of Problem 8. To this end, we need the classical Hilbert spaces

$$
V=\left\{\boldsymbol{v} \in H^{1}\left(\Omega ; \mathbb{R}^{d}\right) \mid \boldsymbol{v}=\mathbf{0} \text { on } \Gamma_{D}\right\}, \quad H=L^{2}\left(\Omega ; \mathbb{R}^{d}\right), \quad \mathcal{H}=L^{2}\left(\Omega ; \mathbb{S}^{d}\right)
$$

with their standard inner products and norms. The symbol $\|\gamma\|$ represents the norm of the trace operator $\gamma: V \rightarrow L^{2}\left(\Gamma ; \mathbb{R}^{d}\right)$. For $\boldsymbol{v} \in H^{1}\left(\Omega ; \mathbb{R}^{d}\right)$, we use the same symbol $\boldsymbol{v}$ for the trace of $\boldsymbol{v}$ on $\Gamma$ and we use the notation $v_{\nu}$ and $\boldsymbol{v}_{\tau}$ for its normal and tangential traces.

For the weak formulation of the problem and further discussion, we need the following hypotheses. $H(\mathscr{A}): \mathscr{A}: \Omega \times \mathbb{S}^{d} \rightarrow \mathbb{S}^{d}$ is such that

(i) $\mathscr{A}(\boldsymbol{x}, \boldsymbol{\varepsilon})=a(\boldsymbol{x}) \varepsilon$ for all $\varepsilon \in \mathbb{S}^{d}$, a.e. $\boldsymbol{x} \in \Omega$.

(ii) $a(\boldsymbol{x})=\left\{a_{i j k l}(\boldsymbol{x})\right\}$ with $a_{i j k l}=a_{j i k l}=a_{l k i j} \in L^{\infty}(\Omega), i, j, k, l=1, \ldots, d$.

(iii) $a_{i j k l}(\boldsymbol{x}) \varepsilon_{i j} \varepsilon_{k l} \geq \alpha\|\varepsilon\|^{2}$ for all $\varepsilon=\left(\varepsilon_{i j}\right) \in \mathbb{S}^{d}$, a.e. $\boldsymbol{x} \in \Omega$ with $\alpha>0$. 
$H(\mathscr{B}): \mathscr{B}: \Omega \times \mathbb{S}^{d} \rightarrow \mathbb{S}^{d}$ is such that

(i) $\mathscr{B}(\boldsymbol{x}, \varepsilon)=b(\boldsymbol{x}) \varepsilon$ for all $\varepsilon \in \mathbb{S}^{d}$, a.e. $\boldsymbol{x} \in \Omega$.

(ii) $b(\boldsymbol{x})=\left\{b_{i j k l}(\boldsymbol{x})\right\}$ with $b_{i j k l}=b_{j i k l}=b_{l k i j} \in L^{\infty}(\Omega), i, j, k, l=1, \ldots, d$.

(iii) $b_{i j k l}(\boldsymbol{x}) \varepsilon_{i j} \varepsilon_{k l} \geq 0$ for all $\varepsilon=\left(\varepsilon_{i j}\right) \in \mathbb{S}^{d}$, a.e. $\boldsymbol{x} \in \Omega$.

$H(\mathscr{C}): \mathscr{C}: Q \times \mathbb{S}^{d} \rightarrow \mathbb{S}^{d}$ is such that

(i) $\mathscr{C}(\boldsymbol{x}, t, \boldsymbol{\varepsilon})=c(\boldsymbol{x}, t) \boldsymbol{\varepsilon}$ for all $\boldsymbol{\varepsilon} \in \mathbb{S}^{d}$, a.e. $(\boldsymbol{x}, t) \in Q$.

(ii) $c(\boldsymbol{x}, t)=\left\{c_{i j k l}(\boldsymbol{x}, t)\right\}$ with $c_{i j k l}=c_{j i k l}=c_{l k i j} \in L^{\infty}(Q), i, j, k, l=1, \ldots, d$.

For the potential function $j_{\nu}$, we assume $H\left(j_{\nu}\right): j_{\nu}: \Gamma_{C} \times \mathbb{R} \rightarrow \mathbb{R}$ is such that

(i) $j_{\nu}(\cdot, r)$ is measurable on $\Gamma_{C}$ for all $r \in \mathbb{R}$ and there is $\bar{e} \in L^{2}\left(\Gamma_{C}\right)$ such that $j_{\nu}(\cdot, \bar{e}(\cdot)) \in L^{1}\left(\Gamma_{C}\right)$.

(ii) $j_{\nu}(\boldsymbol{x}, \cdot)$ is locally Lipschitz on $\mathbb{R}$ for a.e. $\boldsymbol{x} \in \Gamma_{C}$.

(iii) $\left|\partial j_{\nu}(\boldsymbol{x}, r)\right| \leq \bar{c}_{0}$ for all $r \in \mathbb{R}$, a.e. $\boldsymbol{x} \in \Gamma_{C}$ with $\bar{c}_{0} \geq 0$.

(iv) $\left(\partial j_{\nu}\left(\boldsymbol{x}, r_{1}\right)-\partial j_{\nu}\left(\boldsymbol{x}, r_{2}\right)\right)\left(r_{1}-r_{2}\right) \geq-\bar{\beta}\left|r_{1}-r_{2}\right|^{2}$ for all $r_{1}, r_{2} \in \mathbb{R}$, a.e. $\boldsymbol{x} \in \Gamma_{C}$ with $\bar{\beta} \geq 0$.

(v) $j_{\nu}(\boldsymbol{x}, \cdot)$ or $-j_{\nu}(\boldsymbol{x}, \cdot)$ is regular for a.e. $\boldsymbol{x} \in \Gamma_{C}$.

For the damper coefficient $k$ and the friction bound $F_{b}$, we assume $H(k): k: \Gamma_{C} \times \mathbb{R} \rightarrow \mathbb{R}_{+}$is such that

(i) $k(\cdot, r)$ is measurable on $\Gamma_{C}$ for all $r \in \mathbb{R}$.

(ii) there are $k_{1}, k_{2}$ such that $0<k_{1} \leq k(\boldsymbol{x}, r) \leq k_{2}$ for all $r \in \mathbb{R}$, a.e. $\boldsymbol{x} \in \Gamma_{C}$.

(iii) there is $L_{k}>0$ such that $\left|k\left(\boldsymbol{x}, r_{1}\right)-k\left(\boldsymbol{x}, r_{2}\right)\right| \leq L_{k}\left|r_{1}-r_{2}\right|$ for all $r_{1}, r_{2} \in \mathbb{R}$, a.e. $\boldsymbol{x} \in \Gamma_{C}$. $H\left(F_{b}\right): F_{b}: \Gamma_{C} \times \mathbb{R} \rightarrow \mathbb{R}_{+}$is such that

(i) $F_{b}(\cdot, r)$ is measurable on $\Gamma_{C}$ for all $r \in \mathbb{R}$.

(ii) there is $L_{F_{b}}>0$ such that $\left|F_{b}\left(\boldsymbol{x}, r_{1}\right)-F_{b}\left(\boldsymbol{x}, r_{2}\right)\right| \leq L_{F_{b}}\left|r_{1}-r_{2}\right|$ for all $r_{1}, r_{2} \in \mathbb{R}$, a.e. $\boldsymbol{x} \in \Gamma_{C}$.

(iii) $F_{b}(\cdot, 0) \in L^{2}\left(\Gamma_{C}\right)$.

$H\left(f_{0}\right)$ : the densities of body forces, surface tractions and the initial data satisfy

$$
\boldsymbol{f}_{0} \in L^{2}\left(0, T ; L^{2}\left(\Omega ; \mathbb{R}^{d}\right)\right), \quad \boldsymbol{f}_{2} \in L^{2}\left(0, T ; L^{2}\left(\Gamma_{N} ; \mathbb{R}^{d}\right)\right), \quad \boldsymbol{u}_{0}, \boldsymbol{w}_{0} \in V .
$$

Finally, we define $\boldsymbol{f}:(0, T) \rightarrow V^{*}$ by

$$
\langle\boldsymbol{f}(t), \boldsymbol{v}\rangle_{V^{*} \times V}=\left\langle\boldsymbol{f}_{0}(t), \boldsymbol{v}\right\rangle_{H}+\left\langle\boldsymbol{f}_{2}(t), \gamma \boldsymbol{v}\right\rangle_{L^{2}\left(\Gamma_{N} ; \mathbb{R}^{d}\right)}
$$

for all $\boldsymbol{v} \in V$ and a.e. $t \in(0, T)$. Under the above notation, we obtain the following weak formulation of Problem 8 in terms of the displacement.

Problem 9. Find $\boldsymbol{u}:(0, T) \rightarrow V$ such that for all $\boldsymbol{v} \in V$, a.e. $t \in(0, T)$,

$$
\begin{aligned}
\int_{\Omega} \boldsymbol{u}^{\prime \prime}(t) \cdot\left(\boldsymbol{v}-\boldsymbol{u}^{\prime}(t)\right) \mathrm{d} x+\left(\mathscr{A} \varepsilon\left(\boldsymbol{u}^{\prime}(t)\right), \boldsymbol{\varepsilon}\left(\boldsymbol{v}-\boldsymbol{u}^{\prime}(t)\right)\right)_{\mathcal{H}} \\
\quad+\left(\mathscr{B} \varepsilon(\boldsymbol{u}(t)), \boldsymbol{\varepsilon}\left(\boldsymbol{v}-\boldsymbol{u}^{\prime}(t)\right)\right)_{\mathcal{H}}+\left(\int_{0}^{t} \mathscr{C}(t-s) \varepsilon\left(\boldsymbol{u}^{\prime}(s)\right) \mathrm{d} s, \boldsymbol{\varepsilon}\left(\boldsymbol{v}-\boldsymbol{u}^{\prime}(t)\right)\right)_{\mathcal{H}} \\
\quad+\int_{\Gamma_{C}} F_{b}\left(\int_{0}^{t}\left\|\boldsymbol{u}_{\tau}(s)\right\| \mathrm{d} s\right)\left(\left\|\boldsymbol{v}_{\tau}\right\|-\left\|\boldsymbol{u}_{\tau}^{\prime}(t)\right\|\right) \mathrm{d} \Gamma \\
\quad+\int_{\Gamma_{C}} k\left(u_{\nu}(t)\right) j_{\nu}^{0}\left(u_{\nu}^{\prime}(t) ; v_{\nu}-u_{\nu}^{\prime}(t)\right) \mathrm{d} \Gamma \geq\left\langle\boldsymbol{f}(t), \boldsymbol{v}-\boldsymbol{u}^{\prime}(t)\right\rangle_{V^{*} \times V},
\end{aligned}
$$

and

$$
\boldsymbol{u}(0)=\boldsymbol{u}_{0}, \quad \boldsymbol{u}^{\prime}(0)=\boldsymbol{w}_{0}
$$


Let $\boldsymbol{w}=\boldsymbol{u}^{\prime}$. Then

$$
\boldsymbol{u}(t)=\int_{0}^{t} \boldsymbol{w}(s) \mathrm{d} s+\boldsymbol{u}_{0},
$$

and subsequently, $u_{\nu}(t)=\int_{0}^{t} w_{\nu}(s) \mathrm{d} s+u_{0 \nu}$ and $\boldsymbol{u}_{\tau}(t)=\int_{0}^{t} \boldsymbol{w}_{\tau}(s) \mathrm{d} s+\boldsymbol{u}_{0 \tau}$ for $t \in(0, T)$. Using these relations, Problem 9 in terms of velocity can be equivalently formulated as follows.

Problem 10. Find

$\boldsymbol{w}:(0, T) \rightarrow V$ such that for all $\boldsymbol{v} \in V$, a.e. $t \in(0, T)$,

$$
\begin{aligned}
\int_{\Omega} \boldsymbol{w}^{\prime}(t) \cdot(\boldsymbol{v}-\boldsymbol{w}(t)) \mathrm{d} x+(\mathscr{A} \varepsilon(\boldsymbol{w}(t)), \boldsymbol{\varepsilon}(\boldsymbol{v}-\boldsymbol{w}(t)))_{\mathcal{H}} \\
\quad+\left(\mathscr{B} \varepsilon\left(\int_{0}^{t} \boldsymbol{w}(s) \mathrm{d} s+\boldsymbol{u}_{0}\right), \boldsymbol{\varepsilon}(\boldsymbol{v}-\boldsymbol{w}(t))\right)_{\mathcal{H}}+\left(\int_{0}^{t} \mathscr{C}(t-s) \varepsilon(\boldsymbol{w}(s)) \mathrm{d} s, \boldsymbol{\varepsilon}(\boldsymbol{v}-\boldsymbol{w}(t))\right)_{\mathcal{H}} \\
\quad+\int_{\Gamma_{C}} F_{b}\left(\int_{0}^{t}\left\|\int_{0}^{s} \boldsymbol{w}_{\tau}(r) \mathrm{d} r+\boldsymbol{u}_{0 \tau}\right\| \mathrm{d} s\right)\left(\left\|\boldsymbol{v}_{\tau}\right\|-\left\|\boldsymbol{w}_{\tau}(t)\right\|\right) \mathrm{d} \Gamma \\
\quad+\int_{\Gamma_{C}} k\left(\int_{0}^{t} w_{\nu}(s) \mathrm{d} s+u_{0 \nu}\right) j_{\nu}^{0}\left(w_{\nu}(t) ; v_{\nu}-w_{\nu}(t)\right) \mathrm{d} \Gamma \geq\langle\boldsymbol{f}(t), \boldsymbol{v}-\boldsymbol{w}(t)\rangle_{V^{*} \times V},
\end{aligned}
$$

and

$$
\boldsymbol{w}(0)=\boldsymbol{w}_{0} .
$$

Next, let $X=Y=Z=L^{2}\left(\Gamma_{C}\right), U=L^{2}\left(\Gamma_{C} ; \mathbb{R}^{d}\right)$, and introduce operators $A: V \rightarrow V^{*}, R: \mathcal{V} \rightarrow$ $L^{2}(0, T ; Y), R_{1}: \mathcal{V} \rightarrow \mathcal{V}^{*}, S: \mathcal{V} \rightarrow L^{2}(0, T ; Z), M: V \rightarrow X$, and $N: V \rightarrow U$ as follows:

$$
\begin{aligned}
& \langle A \boldsymbol{w}, \boldsymbol{v}\rangle_{V^{*} \times V}=(\mathscr{A} \boldsymbol{\varepsilon}(\boldsymbol{w}), \boldsymbol{\varepsilon}(\boldsymbol{v}))_{\mathcal{H}} \quad \text { for all } \boldsymbol{w}, \boldsymbol{v} \in V, \\
& (R \boldsymbol{w})(t)=\int_{0}^{t}\left\|\int_{0}^{s} \boldsymbol{w}_{\tau}(r) \mathrm{d} r+\boldsymbol{u}_{0 \tau}\right\| \mathrm{d} s \quad \text { for all } \boldsymbol{w} \in \mathcal{V}, t \in(0, T), \\
& \left\langle\left(R_{1} \boldsymbol{w}\right)(t), \boldsymbol{v}\right\rangle_{V^{*} \times V}=\left(\mathscr{B}\left(\int_{0}^{t} \boldsymbol{\varepsilon}(\boldsymbol{w}(s)) \mathrm{d} s+\boldsymbol{u}_{0}\right), \boldsymbol{\varepsilon}(\boldsymbol{v})\right)_{\mathcal{H}} \\
& \quad+\left(\int_{0}^{t} \mathscr{C}(t-s) \varepsilon(\boldsymbol{w}(s)) \mathrm{d} s, \boldsymbol{\varepsilon}(\boldsymbol{v})\right)_{\mathcal{H}} \text { for all } \boldsymbol{w} \in \mathcal{V}, \boldsymbol{v} \in V, t \in(0, T), \\
& (S \boldsymbol{w})(t)=\int_{0}^{t} w_{\nu}(s) \mathrm{d} s+u_{0 \nu} \quad \text { for all } \boldsymbol{w} \in \mathcal{V}, t \in(0, T), \\
& M \boldsymbol{v}=v_{\nu}, \quad N \boldsymbol{v}=\boldsymbol{v}_{\tau} \text { for all } \boldsymbol{v} \in V .
\end{aligned}
$$

Further, consider the boundary potentials $J:(0, T) \times Z \times X \rightarrow \mathbb{R}$ and $\varphi:(0, T) \times Y \times U \rightarrow \mathbb{R}$ defined by

$$
\begin{aligned}
& J(t, z, v)=\int_{\Gamma_{C}} k(z) j_{\nu}(v) \mathrm{d} \Gamma \quad \text { for all } z \in Z, v \in X, t \in(0, T), \\
& \varphi(t, y, \boldsymbol{v})=\int_{\Gamma_{C}} F_{b}(y)\|\boldsymbol{v}\| \mathrm{d} \Gamma \quad \text { for all } y \in Y, \boldsymbol{v} \in U, t \in(0, T) .
\end{aligned}
$$

With the notation above, we formulate the following history-dependent evolution inclusion associated with Problem 10.

Problem 11. Find $\boldsymbol{w} \in \mathcal{W}$ such that

$$
\left\{\begin{array}{c}
\boldsymbol{w}^{\prime}(t)+A \boldsymbol{w}(t)+\left(R_{1} \boldsymbol{w}\right)(t)+M^{*} \partial J(t,(S \boldsymbol{w})(t), M \boldsymbol{w}(t)) \\
\quad+N^{*} \partial \varphi(t,(R \boldsymbol{w})(t), N \boldsymbol{w}(t)) \ni \boldsymbol{f}(t) \text { a.e. } t \in(0, T) \\
\boldsymbol{w}(0)=\boldsymbol{w}_{0}
\end{array}\right.
$$


The following result concerns the well-posedness of Problem 11.

Theorem 12. Assume hypotheses $H(\mathscr{A}), H(\mathscr{B}), H(\mathscr{C}), H\left(j_{\nu}\right), H(k), H\left(F_{b}\right)$, and $H\left(f_{0}\right)$. If

$$
\alpha>\bar{\beta} k_{2}\|\gamma\|^{2},
$$

then Problem 11 has a unique solution $\boldsymbol{w} \in \mathcal{W}$. Moreover, the map $\mathcal{V}^{*} \times V \ni\left(\boldsymbol{f}, \boldsymbol{w}_{0}\right) \mapsto \boldsymbol{w} \in \mathcal{W}$ is continuous in weak topologies.

Proof. We will apply Theorem 7 with a suitable choice of spaces, operators and functionals introduced above. First, it is clear that under $H(\mathscr{A})$ the operator $A$ defined by (5.11) satisfies $A \in \mathcal{L}\left(V, V^{*}\right)$, and $H(A)_{1}$ with $a_{0}=0, a_{1}=\|A\|_{\mathcal{L}\left(V, V^{*}\right)}$ and $m_{A}=\alpha$.

Now, we verify properties in $H(J)_{1}$ for the functional $J$ given by (5.16). Conditions $H(J)(\mathrm{i})-$ (iii) follow from $H\left(j_{\nu}\right)(\mathrm{i})-(\mathrm{iii}), H(k)(\mathrm{i})$, (ii) and [19, Theorem 3.47(i)-(iii)]. From [19, Proposition 3.37(ii), Theorem 3.47(v), (vi)], we have

$$
\partial J(t, z, v) \subset \int_{\Gamma_{C}} k(z) \partial j_{\nu}(v) \mathrm{d} \Gamma \text { for all } z \in Z, v \in X \text {, a.e. } t \in(0, T) .
$$

Hence, we obtain that $H(J)$ (iv) holds with $c_{0 J}(t)=k_{2} \bar{c}_{0} \sqrt{\left|\Gamma_{C}\right|}$, and $c_{1 J}=c_{2 J}=0$. Moreover, by a similar argument as in [8, Theorem 13], we get

$$
\begin{aligned}
& J^{0}\left(t, z_{1}, v_{1} ; v_{2}-v_{1}\right)+J^{0}\left(t, z_{2}, v_{2} ; v_{1}-v_{2}\right) \\
& \quad \leq m_{J}\left\|v_{1}-v_{2}\right\|_{X}^{2}+\bar{m}_{J}\left\|z_{1}-z_{2}\right\|_{Z}\left\|v_{1}-v_{2}\right\|_{X}
\end{aligned}
$$

for all $z_{1}, z_{2} \in Z, v_{1}, v_{2} \in X$, a.e. $t \in(0, T)$, where $m_{J}=\bar{\beta} k_{2}$ and $\bar{m}_{J}=\bar{c}_{0} L_{k}$. Hence, $H(J)(\mathrm{v})$ follows.

Next, we verify $H(J)_{1}(\mathrm{vi})$. Let $\left\{z_{n}\right\} \subset Z,\left\{v_{n}\right\} \subset X, z_{n} \rightarrow z$ in $Z$ and $v_{n} \rightarrow v$ in $X$. By passing to a subsequence, we may suppose that $z_{n}(x) \rightarrow z(x)$ and $v_{n}(x) \rightarrow v(x)$ for a.e. $x \in \Gamma_{C}$. Then, we use properties of the generalized directional derivative and generalized gradient stated in [19, Proposition 3.23(ii), (iii), Theorem 3.47(iv)]. By $H\left(j_{\nu}\right)$ and $H(k)$, for all $w \in X$ and a.e. $t \in(0, T)$, we obtain

$$
\begin{aligned}
& J^{0}\left(t, z_{n}, v_{n} ; w\right) \leq \int_{\Gamma_{C}} k\left(z_{n}(x)\right) j_{\nu}^{0}\left(v_{n}(x) ; w(x)\right) \mathrm{d} \Gamma \\
& \quad \leq L_{k} \bar{c}_{0} \int_{\Gamma_{C}}\left|z_{n}(x)-z(x)\right||w(x)| \mathrm{d} \Gamma+\int_{\Gamma_{C}} k(z(x)) j_{\nu}^{0}\left(v_{n}(x) ; w(x)\right) \mathrm{d} \Gamma
\end{aligned}
$$

which entails

$$
\begin{aligned}
& \limsup J^{0}\left(t, z_{n}, v_{n} ; w\right) \leq \limsup \int_{\Gamma_{C}} k(z(x)) j_{\nu}^{0}\left(v_{n}(x) ; w(x)\right) \mathrm{d} \Gamma \\
& \quad \leq \int_{\Gamma_{C}} k(z(x)) j_{\nu}^{0}(v(x) ; w(x)) \mathrm{d} \Gamma=J^{0}(t, z, v ; w) .
\end{aligned}
$$

The last inequality follows from hypothesis $H\left(j_{\nu}\right)(\mathrm{v})$ and [19, Theorem 3.47(vii)]. Hence, we deduce that $J^{0}(t, \cdot, \cdot ; w): Z \times X \rightarrow \mathbb{R}$ is upper semicontinuous for all $w \in X$, a.e. $t \in(0, T)$. Condition $H(J)_{1}(\mathrm{vi})$ is verified.

Subsequently, by a modification of the reasoning used in [8, Theorem 13], we easily verify that $\varphi$ satisfies conditions $H(\varphi)$ (ii)-(iv) with $c_{0 \varphi}(t)=\sqrt{2}\left\|F_{b}(0)\right\|_{Y}, c_{1 \varphi}=\sqrt{2} L_{F_{b}}$ and $c_{2 \varphi}=0$. Further, a direct calculation implies that $H(\varphi)(\mathrm{v})$ holds with $\beta_{\varphi}=L_{F_{b}}$. We conclude that condition $H(\varphi)$ is satisfied.

By definition (5.15), it is clear that operators $M$ and $N$ satisfy $H(M, N)$ and they are compact. From [21, Theorem 2.18], we infer that the Nemytskii operators corresponding to $M$, and $N$ are compact too. Therefore, condition $H(M, N)_{1}$ holds.

Next, by hypothesis $H\left(f_{0}\right)$ and definition (5.8), we know that the regularity condition $\left(H_{0}\right)$ holds. Hypothesis $\left(H_{3}\right)$ is a consequence of the smallness condition (5.18).

We now verify condition $\left(H_{4}\right)$. It follows from [33] that operators $R, R_{1}$ and $S$ given by (5.12)(5.14), under hypotheses $H(\mathscr{B})$ and $H(\mathscr{C})$, are history-dependent with constants $c_{R}=T\|\gamma\|, c_{R_{1}}=$ 
$\|\mathscr{B}\|+\|\mathscr{C}\|_{L^{\infty}\left(Q ; \mathbb{S}^{d}\right)}$ and $c_{S}=\|\gamma\|$. Hence, $\left(H_{2}\right)$ follows. Next, to prove compactness of operators $R$ and $S$. We present the proof for $R$, while a proof for $S$ is omitted being analogous. Let $\boldsymbol{v}_{n} \rightarrow \boldsymbol{v}$ weakly in $\mathcal{W}$. From $\left[21\right.$, Theorem 2.18], we know that $\gamma \boldsymbol{v}_{n} \rightarrow \gamma \boldsymbol{v}$ in $L^{2}\left(0, T ; L^{2}\left(\Gamma_{C} ; \mathbb{R}^{d}\right)\right)$, where $\gamma: \mathcal{V} \rightarrow L^{2}\left(0, T ; L^{2}\left(\Gamma_{C} ; \mathbb{R}^{d}\right)\right)$ is the Nemytskii operator corresponding to the trace $\gamma$ (for simplicity denoted in the same way). By Hölder's inequality and a direct calculation, we have

$$
\begin{aligned}
& \left\|\left(R \boldsymbol{v}_{n}\right)(t)-(R \boldsymbol{v})(t)\right\|_{Y} \leq T \int_{0}^{t}\left\|\boldsymbol{v}_{n}(s)-\boldsymbol{v}(s)\right\|_{L^{2}\left(\Gamma_{C} ; \mathbb{R}^{d}\right)} \mathrm{d} s \\
& \quad \leq T \sqrt{T}\left\|\boldsymbol{v}_{n}-\boldsymbol{v}\right\|_{L^{2}\left(0, T ; L^{2}\left(\Gamma_{C} ; \mathbb{R}^{d}\right)\right)}
\end{aligned}
$$

for a.e. $t \in(0, T)$. Hence, it follows $\left(R \boldsymbol{v}_{n}\right)(t) \rightarrow(R \boldsymbol{v})(t)$ in $Y$, for a.e. $t \in(0, T)$. By the Lebesgue dominated convergence theorem, we get $R \boldsymbol{v}_{n} \rightarrow R \boldsymbol{v}$ in $L^{2}(0, T ; Y)$. Thus, operator $R: \mathcal{W} \subset \mathcal{V} \rightarrow L^{2}(0, T ; Y)$ is compact. It is also clear that $(R 0, S 0)$ belongs to a bounded subset of $L^{2}(0, T ; Y \times Z)$.

To prove the continuity of $R_{1}$ in weak topologies, we denote

$$
\begin{aligned}
\left\langle\left(R_{11} \boldsymbol{w}\right)(t), \boldsymbol{v}\right\rangle_{V^{*} \times V} & =\left(\mathscr{B}\left(\int_{0}^{t} \boldsymbol{\varepsilon}(\boldsymbol{w}(s)) \mathrm{d} s+\boldsymbol{u}_{0}\right), \boldsymbol{\varepsilon}(\boldsymbol{v})\right)_{\mathcal{H}}, \\
\left\langle\left(R_{12} \boldsymbol{w}\right)(t), \boldsymbol{v}\right\rangle_{V^{*} \times V} & =\left(\int_{0}^{t} \mathscr{C}(t-s) \boldsymbol{\varepsilon}(\boldsymbol{w}(s)) \mathrm{d} s, \boldsymbol{\varepsilon}(\boldsymbol{v})\right)_{\mathcal{H}}
\end{aligned}
$$

for all $\boldsymbol{w} \in \mathcal{V}, \boldsymbol{v} \in V, t \in(0, T)$. Let $\left\{\boldsymbol{v}_{n}\right\} \subset \mathcal{V}$ be such that $\boldsymbol{v}_{n} \rightarrow \boldsymbol{v}$ weakly in $\mathcal{V}$. Then, for all $\psi \in V^{*}$, all $t \in[0, T]$, we have

$$
\begin{aligned}
& \left\langle\int_{0}^{t} \boldsymbol{v}_{n}(s) \mathrm{d} s, \psi\right\rangle_{V^{*} \times V}=\int_{0}^{t}\left\langle\boldsymbol{v}_{n}(s), \psi\right\rangle_{V^{*} \times V} \mathrm{~d} s=\left\langle\boldsymbol{v}_{n}, \psi\right\rangle_{\mathcal{V}^{*} \times \mathcal{V}} \\
& \rightarrow\langle\boldsymbol{v}, \psi\rangle_{\mathcal{V}^{*} \times \mathcal{V}}=\int_{0}^{t}\langle\boldsymbol{v}(s), \psi\rangle_{V^{*} \times V} \mathrm{~d} s=\left\langle\int_{0}^{t} \boldsymbol{v}(s) \mathrm{d} s, \psi\right\rangle_{V^{*} \times V}
\end{aligned}
$$

that is,

$$
\int_{0}^{t} \boldsymbol{v}_{n}(s) \mathrm{d} s+\boldsymbol{u}_{0} \rightarrow \int_{0}^{t} \boldsymbol{v}(s) \mathrm{d} s+\boldsymbol{u}_{0} \text { weakly in } V, \text { for all } t \in[0, T] .
$$

Since $\mathcal{B}$ is linear and continuous, we deduce $R_{11} \boldsymbol{v}_{n} \rightarrow R_{11} \boldsymbol{v}$ weakly in $\mathcal{V}^{*}$. Also since $R_{12}$ is linear and continuous, it is also weakly-weakly continuous, and therefore, $R_{12} \boldsymbol{v}_{n} \rightarrow R_{12} \boldsymbol{v}$ weakly in $\mathcal{V}^{*}$. We conclude that $R_{1}$ is weakly-weakly continuous, history-dependent, and clearly, $R_{1} 0$ is bounded in $L^{2}\left(0, T ; V^{*}\right)$. In this way, condition $\left(H_{4}\right)$ is verified. The conclusion of the theorem follows now from Theorem 7 , which completes the proof.

Observe that Problems 10 and 11 are equivalent. This follows form the facts that every solution to Problem 11 is a solution to Problem 10, and that both problems have unique solutions. Thus, $\boldsymbol{w} \in \mathcal{W}$ solves inequality in Problem 10 if and only if it is solves inclusion in Problem 11. We apply Theorem 12 to deduce the following well-posedness result for variational-hemivariational inequality in Problem 9. It shows the continuous dependence of the solution to the contact problem with respect to the densities of applied forces and the initial data.

Corollary 13. Assume hypotheses $H(\mathscr{A}), H(\mathscr{B}), H(\mathscr{C}), H\left(j_{\nu}\right), H(k), H\left(F_{b}\right), H\left(f_{0}\right)$, and (5.18). Then, Problem 9 has a unique solution with regularity $\boldsymbol{u} \in \mathcal{V}$ and $\boldsymbol{u}^{\prime} \in \mathcal{W}$. Moreover, if $\left\{\left(\boldsymbol{f}_{0}^{n}, \boldsymbol{f}_{N}^{n}, \boldsymbol{u}_{0}^{n}, \boldsymbol{w}_{0}^{n}\right)\right\} \subset$ $L^{2}\left(0, T ; L^{2}\left(\Omega ; \mathbb{R}^{d}\right) \times L^{2}\left(\Gamma_{N} ; \mathbb{R}^{d}\right)\right) \times V \times V$, and

$$
\begin{aligned}
& \left(\boldsymbol{f}_{0}^{n}, \boldsymbol{f}_{N}^{n}\right) \rightarrow\left(\boldsymbol{f}_{0}, \boldsymbol{f}_{N}\right) \text { weakly in } L^{2}\left(0, T ; L^{2}\left(\Omega ; \mathbb{R}^{d}\right) \times L^{2}\left(\Gamma_{N} ; \mathbb{R}^{d}\right)\right), \\
& \left(\boldsymbol{u}_{0}^{n}, \boldsymbol{w}_{0}^{n}\right) \rightarrow\left(\boldsymbol{u}_{0}, \boldsymbol{w}_{0}\right) \text { weakly in } V \times V,
\end{aligned}
$$


then

$$
\begin{aligned}
& \boldsymbol{u}_{n}(t) \rightarrow \boldsymbol{u}(t) \text { weakly in } V, \text { for all } t \in[0, T], \\
& \boldsymbol{u}_{n}^{\prime} \rightarrow \boldsymbol{u}^{\prime} \text { weakly in } \mathcal{W},
\end{aligned}
$$

where $\left\{\boldsymbol{u}_{n}\right\}$ and $\boldsymbol{u}$ are unique solutions to Problem 9 corresponding to $\left(\boldsymbol{f}_{0}^{n}, \boldsymbol{f}_{N}^{n}, \boldsymbol{u}_{0}^{n}, \boldsymbol{w}_{0}^{n}\right)$ and $\left(\boldsymbol{f}_{0}, \boldsymbol{f}_{N}, \boldsymbol{u}_{0}\right.$, $\left.\boldsymbol{w}_{0}\right)$, respectively.

Proof. Assume (5.20) and (5.21). Let $\boldsymbol{f}_{n}, \boldsymbol{f} \in \mathcal{V}^{*}$ be elements defined by (5.9) corresponding to $\left(\boldsymbol{f}_{0}^{n}, \boldsymbol{f}_{N}^{n}\right)$ and $\left(\boldsymbol{f}_{0}, \boldsymbol{f}_{N}\right)$, respectively. Since the map $\left(\boldsymbol{f}_{0}, \boldsymbol{f}_{n}\right) \mapsto \boldsymbol{f}$ is linear and continuous, we have $\boldsymbol{f}_{n} \rightarrow \boldsymbol{f}$ weakly in $\mathcal{V}^{*}$. Combining this with hypothesis $\boldsymbol{w}_{0}^{n} \rightarrow \boldsymbol{w}_{0}$ weakly in $V$, by Theorem 12 , we infer $\boldsymbol{w}_{n} \rightarrow \boldsymbol{w}$ weakly in $\mathcal{W}$, where $\boldsymbol{u}_{n}(t)=\int_{0}^{t} \boldsymbol{w}_{n}(s) \mathrm{d} s+\boldsymbol{u}_{0}^{n}$ and $\boldsymbol{u}(t)=\int_{0}^{t} \boldsymbol{w}(s) \mathrm{d} s+\boldsymbol{u}_{0}$ for all $t \in[0, T]$, see (5.10).

Clearly, we have $\boldsymbol{u}_{n}^{\prime} \rightarrow \boldsymbol{u}^{\prime}$ weakly in $\mathcal{W}$, and analogously as in the proof of (5.19), we obtain $\int_{0}^{t} \boldsymbol{w}_{n}(s) \mathrm{d} s \rightarrow \int_{0}^{t} \boldsymbol{w}(s) \mathrm{d} s$ weakly in $V$, for all $t \in[0, T]$. The latter together with hypothesis (5.21) implies (5.22). This completes the proof.

\section{Application to a semipermeability problem}

In this section, we illustrate the applicability of results in Sect. 4 in analysis of a semipermeability problem. Our aim is to provide the weak formulation of the problem which will be a variational-hemivariational inequality without history-dependent operators and to establish its well-posedness.

The semipermeability boundary conditions describe behavior of various types of membranes, natural and artificial ones and arise in models of heat conduction, electrostatics, hydraulics and in the description of flow of Bingham's fluids, where the solution represents temperature, electric potential and pressure. These boundary conditions were first examined by Duvaut and Lions [5] in the convex setting, where semipermeability relations were assumed to be monotone and they led to variational inequalities. More generally, nonmonotone semipermeability conditions can be modeled by the Clarke generalized gradient, see, e.g., $[6,15,25,26]$ and the references therein.

Let $\Omega$ be a bounded domain in $\mathbb{R}^{d}$ with Lipschitz continuous boundary $\Gamma$. The latter is decomposed into three mutually disjoint and relatively open subsets $\Gamma_{a}, \Gamma_{b}$ and $\Gamma_{c}$ of $\Gamma$ such that $\Gamma=\bar{\Gamma}_{a} \cup \bar{\Gamma}_{b} \cup \bar{\Gamma}_{c}$ and $m\left(\Gamma_{c}\right)>0$. We denote $Q=\Omega \times(0, T), \Sigma_{a}=\Gamma_{a} \times(0, T), \Sigma_{b}=\Gamma_{b} \times(0, T)$ and $\Sigma_{c}=\Gamma_{c} \times(0, T)$ with $0<T<\infty$. Consider the following initial-boundary value problem.

Problem 14. Find $u=u(x, t)$ such that

$$
\begin{array}{r}
\frac{\partial u}{\partial t}+A u+\partial j_{1}(u)+\partial g_{1}(u) \ni f_{1} \text { in } Q \\
\frac{\partial u}{\partial \nu_{A}}+\partial j_{2}(u) \ni f_{a} \text { on } \Sigma_{a} \\
\frac{\partial u}{\partial \nu_{A}}+\partial g_{2}(u) \ni f_{b} \text { on } \Sigma_{b} \\
u=0 \text { on } \Sigma_{c} \\
u(0)=u_{0} \text { in } \Omega .
\end{array}
$$

where $A$ represents a linear operator $A: V \rightarrow V^{*}, \frac{\partial u}{\partial \nu_{A}}$ denotes the conormal derivative with respect to operator $A$, and $\nu$ stands for the unit outward normal on the boundary. Problem 14 has been studied in [6] under more restrictive hypotheses and a different weak formulation.

To provide the weak formulation of Problem (14), we introduce assumptions on the data of the problem. Let $V=\left\{v \in H^{1}(\Omega) \mid v=0\right.$ on $\left.\Gamma_{c}\right\}, H=L^{2}(\Omega), \mathcal{V}=L^{2}(0, T ; V), \mathcal{W}=\left\{u \in \mathcal{V} \mid u^{\prime} \in \mathcal{V}^{*}\right\}$. We denote by $i: V \rightarrow H$ the embedding operator and by $\gamma: V \rightarrow L^{2}(\Gamma)$ the trace operator. For $v \in H^{1}(\Omega)$, we always write $v$ instead of $i v$ and $\gamma v$.

We need the following hypotheses on the data. 
$\underline{H(A)_{2}}: A: V \rightarrow V^{*}$ is such that $A=-\sum_{i, j=1}^{d} D_{i}\left(a_{i j}(x) D_{j}\right)$, and

(i) $a_{i j} \in L^{\infty}(\Omega)$ for $i, j=1, \ldots, d$.

(ii) $\sum_{i, j=1}^{d} a_{i j}(x) \xi_{i} \xi_{j} \geq \alpha\|\xi\|^{2}$ for all $\xi \in \mathbb{R}^{d}$, a.e. $x \in \Omega$ with $\alpha>0$.

$H\left(j_{1}\right): j_{1}: Q \times \mathbb{R} \rightarrow \mathbb{R}$ is such that

(i) $j_{1}(\cdot, \cdot, r)$ is measurable on $Q$ for all $r \in \mathbb{R}$ and there exists $e_{1} \in L^{2}(\Omega)$ such that $j_{1}\left(\cdot, \cdot, e_{1}(\cdot)\right) \in L^{1}(Q)$.

(ii) $j_{1}(x, t, \cdot)$ is locally Lipschitz for a.e. $(x, t) \in Q$.

(iii) $\left|\partial j_{1}(\boldsymbol{x}, t, r)\right| \leq c_{0 j}(t)+c_{1 j}|r|$ for all $r \in \mathbb{R}$, a.e. $(\boldsymbol{x}, t) \in Q$ with $c_{0 j} \in L_{+}^{2}(0, T), c_{1 j} \geq 0$.

(iv) $\left(\partial j_{1}\left(\boldsymbol{x}, t, r_{1}\right)-\partial j_{1}\left(\boldsymbol{x}, t, r_{2}\right)\right)\left(r_{1}-r_{2}\right) \geq-\beta_{1 j}\left|r_{1}-r_{2}\right|^{2}$ all $r_{1}, r_{2} \in \mathbb{R}$, a.e. $(\boldsymbol{x}, t) \in Q$ with $\beta_{1 j} \geq 0$.

$H\left(j_{2}\right): j_{2}: \Sigma_{a} \times \mathbb{R} \rightarrow \mathbb{R}$ is such that

(i) $j_{2}(\cdot, \cdot, r)$ is measurable on $\Sigma_{a}$ for all $r \in \mathbb{R}$ and there exists $e_{2} \in L^{2}\left(\Gamma_{a}\right)$ such that $j_{2}\left(\cdot, \cdot, e_{2}(\cdot)\right) \in$ $L^{1}\left(\Sigma_{a}\right)$.

(ii) $j_{2}(x, t, \cdot)$ is locally Lipschitz for a.e. $(x, t) \in \Sigma_{a}$.

(iii) $\left|\partial j_{2}(\boldsymbol{x}, t, r)\right| \leq c_{2 j}(t)+c_{3 j}|r|$ for all $r \in \mathbb{R}$, a.e. $(\boldsymbol{x}, t) \in \Sigma_{a}$ with $c_{2 j} \in L_{+}^{2}(0, T), c_{3 j} \geq 0$.

(iv) $\left(\partial j_{2}\left(\boldsymbol{x}, t, r_{1}\right)-\partial j_{2}\left(\boldsymbol{x}, t, r_{2}\right)\right)\left(r_{1}-r_{2}\right) \geq-\beta_{2 j}\left|r_{1}-r_{2}\right|^{2}$ all $r_{1}, r_{2} \in \mathbb{R}$, a.e. $(\boldsymbol{x}, t) \in \Sigma_{a}$ with $\beta_{2 j} \geq 0$.

$H\left(g_{1}\right): g_{1}: Q \times \mathbb{R} \rightarrow \mathbb{R}$ is such that

(i) $g_{1}(\cdot, \cdot, r)$ is measurable on $Q$ for all $r \in \mathbb{R}$.

(ii) $g_{1}(x, t, \cdot)$ is convex and l.s.c. for a.e. $(x, t) \in Q$.

(iii) $\left|\partial g_{1}(\boldsymbol{x}, t, r)\right| \leq c_{0 g}(t)+c_{1 g}|r|$ for all $r \in \mathbb{R}$, a.e. $(\boldsymbol{x}, t) \in Q$ with $c_{0 g} \in L_{+}^{2}(0, T), c_{1 g} \geq 0$.

$H\left(g_{2}\right): g_{2}: \Sigma_{b} \times \mathbb{R} \rightarrow \mathbb{R}$ is such that

(i) $g_{2}(\cdot, \cdot, r)$ is measurable on $\Sigma_{b}$ for all $r \in \mathbb{R}$.

(ii) $g_{2}(x, t, \cdot)$ is convex and l.s.c. for a.e. $(x, t) \in \Sigma_{b}$.

(iii) $\left|\partial g_{2}(\boldsymbol{x}, t, r)\right| \leq c_{2 g}(t)+c_{3 g}|r|$ for all $r \in \mathbb{R}$, a.e. $(\boldsymbol{x}, t) \in \Sigma_{b}$ with $c_{2 g} \in L_{+}^{2}(0, T), c_{3 g} \geq 0$.

$H(f): f_{1} \in L^{2}(Q), f_{a} \in L^{2}\left(\Sigma_{a}\right), f_{b} \in L^{2}\left(\Sigma_{b}\right), u_{0} \in V$.

$\overline{\left(H_{5}\right):} \alpha>\beta_{1 j}\|i\|^{2}+\beta_{2 j}\|\gamma\|^{2}$.

By a standard procedure, we obtain the following weak formulation of Problem (14).

Problem 15. Find $u \in \mathcal{W}$ such that for all $v \in V$, a.e. $t \in(0, T)$, we have

$$
\begin{aligned}
& \left\langle u^{\prime}(t)+A u(t)-f(t), v-u(t)\right\rangle_{V^{*} \times V} \\
& \quad+\int_{\Omega} j_{1}^{0}(x, t, u(t) ; v-u(t)) \mathrm{d} x+\int_{\Gamma_{a}} j_{2}^{0}(x, t, u(t) ; v-u(t)) \mathrm{d} \Gamma \\
& \quad+\int_{\Omega}\left(g_{1}(x, t, v)-g_{1}(x, t, u(t))\right) \mathrm{d} x+\int_{\Gamma_{b}}\left(g_{2}(x, t, v)-g_{2}(x, t, u(t))\right) \mathrm{d} \Gamma \geq 0, \\
& u(0)=u_{0} .
\end{aligned}
$$

Here, $f:(0, T) \rightarrow V^{*}$ is given by

$$
\langle f(t), v\rangle_{V^{*} \times V}=\int_{\Omega} f_{1}(t) v \mathrm{~d} x+\int_{\Gamma_{a}} f_{a}(t) v \mathrm{~d} \Gamma+\int_{\Gamma_{b}} f_{b}(t) v \mathrm{~d} \Gamma
$$

for $v \in V$, a.e. $t \in(0, T)$.

Theorem 16. Assume hypotheses $H(A)_{2}, H\left(j_{1}\right), H\left(j_{2}\right), H\left(g_{1}\right), H\left(g_{2}\right), H(f)$, and $\left(H_{5}\right)$. Then, Problem 15 has a unique solution $u \in \mathcal{W}$ and the map

$$
L^{2}(Q) \times L^{2}\left(\Sigma_{a}\right) \times L^{2}\left(\Sigma_{b}\right) \times V \ni\left(f_{1}, f_{a}, f_{b}, u_{0}\right) \mapsto u \in \mathcal{W}
$$

is continuous, where all spaces are endowed with their weak topologies. 
Proof. We sketch the main points of the proof. We introduce functionals $J_{1}:(0, T) \times H \rightarrow \mathbb{R}, J_{2}:(0, T) \times$ $L^{2}\left(\Gamma_{a}\right) \rightarrow \mathbb{R}, \varphi_{1}:(0, T) \times H \rightarrow \mathbb{R}$, and $\varphi_{2}:(0, T) \times L^{2}\left(\Gamma_{b}\right) \rightarrow \mathbb{R}$ defined by

$$
\begin{aligned}
& J_{1}(t, v)=\int_{\Omega} j_{1}(x, t, v(x)) \mathrm{d} x \text { for } v \in H, \\
& J_{2}(t, v)=\int_{\Gamma_{a}} j_{2}(x, t, v(x)) \mathrm{d} x \text { for } v \in L^{2}\left(\Gamma_{a}\right), \\
& \varphi_{1}(t, v)=\int_{\Omega} g_{1}(x, t, v(x)) \mathrm{d} x \text { for } v \in H, \\
& \varphi_{2}(t, v)=\int_{\Gamma_{b}} g_{2}(x, t, v(x)) \mathrm{d} x \text { for } v \in L^{2}\left(\Gamma_{b}\right),
\end{aligned}
$$

and operators $M_{1}=N_{1}=i \in \mathcal{L}(V, H), M_{2}=N_{2}=\gamma \in \mathcal{L}\left(V, L^{2}(\Gamma)\right)$. With this notation, we consider the following evolution inclusion.

Problem 17. Find $u \in \mathcal{W}$ such that

$$
\left\{\begin{array}{l}
u^{\prime}(t)+A u(t)+M_{1}^{*} \partial J_{1}\left(t, M_{1} u(t)\right)+M_{2}^{*} \partial J_{2}\left(t, M_{2} u(t)\right) \\
\quad+N_{1}^{*} \partial \varphi_{1}\left(t, N_{1} u(t)\right)+N_{2}^{*} \partial \varphi_{2}\left(t, N_{2} u(t)\right) \ni f(t) \text { a.e. } t \in(0, T), \\
u(0)=u_{0} .
\end{array}\right.
$$

It is clear by definitions of the convex and Clarke subdifferentials, and properties of the generalized directional derivative, see [19, Theorem 3.47], that any solution to Problem 17 is also a solution to Problem 15. We will show below that the solution to Problem 11 is unique, and moreover, by a direct calculation we verify that under our hypotheses the solution to Problem 15 is also unique. Hence, we conclude that Problems 15 and 17 are equivalent.

Problem 11 is now treated by exploiting the methods used in Theorems 5 and 7 . Since the operator $A \in \mathcal{L}\left(V, V^{*}\right)$ is coercive, it satisfies condition $H(A)_{1}$. Note that $J_{1}$ satisfies condition $H(J)$, where the variable $z$ is omitted, $X=H, m_{J}=\beta_{1 j}$, and $\bar{m}_{J}=0$. Taking into account that $J_{1}^{0}(t, \cdot ; w): X \rightarrow \mathbb{R}$ is upper semicontinuous for all $w \in H$, a.e. $t \in(0, T)$, see [19, Proposition 3.23(ii)], we deduce that $H(J)_{1}$ holds too. Analogously, we check that $J_{2}$ satisfies $H(J)_{1}$ with $m_{J}=\beta_{2 j}$, and $\bar{m}_{J}=0$.

Furthermore, we easily verify that both $\varphi_{1}$ and $\varphi_{2}$ satisfy condition $H(\varphi)$. In particular, $H(\varphi)(\mathrm{v})$ holds automatically. The Nemytskii operators corresponding to $M_{1}$ and $M_{2}$ are compact, see [6, Examples 5.2 and 5.3]. Hence, $H(M, N)_{1}$ is satisfied. Conditions $\left(H_{2}\right)$ and $\left(H_{4}\right)$ hold trivially. Condition $\left(H_{5}\right)$ implies the smallness assumption of type $\left(H_{3}\right)$. We are in a position to apply Theorems 5 and 7 to Problem 17 to obtain its unique solvability and a continuous dependence result. Since Problems 15 and 17 are equivalent with their unique solution, this completes the proof.

Open Access. This article is distributed under the terms of the Creative Commons Attribution 4.0 International License (http://creativecommons.org/licenses/by/4.0/), which permits unrestricted use, distribution, and reproduction in any medium, provided you give appropriate credit to the original author(s) and the source, provide a link to the Creative Commons license, and indicate if changes were made.

Publisher's Note Springer Nature remains neutral with regard to jurisdictional claims in published maps and institutional affiliations.

\section{References}

[1] Aubin, J.P., Cellina, A.: Differential Inclusions: Set-Valued Maps and Viability Theory. Springer, Berlin (1984)

[2] Clarke, F.H.: Optimization and Nonsmooth Analysis. Wiley, New York (1983)

[3] Denkowski, Z., Migórski, S., Papageorgiou, N.S.: An Introduction to Nonlinear Analysis: Theory. Kluwer Academic/Plenum Publishers, Dordrecht (2003) 
[4] Denkowski, Z., Migórski, S., Papageorgiou, N.S.: An Introduction to Nonlinear Analysis: Applications. Kluwer Academic/Plenum Publishers, Dordrecht (2003)

[5] Duvaut, G., Lions, J.L.: Inequalities in Mechanics and Physics. Springer, Berlin (1976)

[6] Gasinski, L., Migórski, S., Ochal, A.: Existence results for evolutionary inclusions and variational-hemivariational inequalities. Appl. Anal. 94, 1670-1694 (2014)

[7] Han, W., Migórski, S., Sofonea, M.: A class of variational-hemivariational inequalities with applications to frictional contact problems. SIAM J. Math. Anal. 46, 3891-3912 (2014)

[8] Han, W., Migórski, S., Sofonea, M.: Analysis of a general dynamic history-dependent variational-hemivariational inequality. Nonlinear Anal. RWA 36, 69-88 (2017)

[9] Kulig, A., Migórski, S.: Solvability and continuous dependence results for second order nonlinear inclusion with Volterratype operator. Nonlinear Anal. 75, 4729-4746 (2012)

[10] Liu, Z., Migórski, S., Zeng, S.: Partial differential variational inequalities involving nonlocal boundary conditions in Banach spaces. J. Differ. Equ. 263, 3989-4006 (2017)

[11] Liu, Z., Zeng, S., Motreanu, D.: Evolutionary problems driven by variational inequalities. J. Differ. Equ. 260, 6787-6799 (2016)

[12] Liu, Z., Zeng, S., Motreanu, D.: Partial differential hemivariational inequalities. Adv. Nonlinear Anal. 7, 571-586 (2018)

[13] Migórski, S.: On existence of solutions for parabolic hemivariational inequalities. J. Comput. Appl. Math. 129, 77-87 (2001)

[14] Migórski, S.: Existence of solutions for a class of history-dependent evolution hemivariational inequalities. Dyn. Syst. Appl. 21, 319-330 (2012)

[15] Migórski, S., Ochal, A.: Boundary hemivariational inequality of parabolic type. Nonlinear Anal. 57, 579-596 (2004)

[16] Migórski, S., Ochal, A.: Quasi-static hemivariational inequality via vanishing acceleration approach. SIAM J. Math. Anal. 41, 1415-1435 (2009)

[17] Migórski, S., Ochal, A., Sofonea, M.: Integrodifferential hemivariational inequalities with applications to viscoelastic frictional contact. Math. Models Methods Appl. Sci. 18, 271-290 (2008)

[18] Migórski, S., Ochal, A., Sofonea, M.: History-dependent subdifferential inclusions and hemivariational inequalities in contact mechanics. Nonlinear Anal. RWA 12, 3384-3396 (2011)

[19] Migórski, S., Ochal, A., Sofonea, M.: Nonlinear Inclusions and Hemivariational Inequalities. Models and Analysis of Contact Problems. Advances in Mechanics and Mathematics, vol. 26. Springer, New York (2013)

[20] Migórski, S., Ochal, A., Sofonea, M.: History-dependent variational-hemivariational inequalities in contact mechanics. Nonlinear Anal. RWA 22, 604-618 (2015)

[21] Migórski, S., Ochal, A., Sofonea, M.: Evolutionary inclusions and hemivariational inequalities, Chapter 2. In: Han, W., et al. (eds.) Advances in Variational and Hemivariational Inequalities: Theory, Numerical Analysis, and Applications. Advances in Mechanics and Mathematics Series, vol. 33, pp. 39-64. Springer, Cham (2015)

[22] Migórski, S., Ogorzaly, J.: A class of evolution variational inequalities with memory and its application to viscoelastic frictional contact problems. J. Math. Anal. Appl. 442, 685-702 (2016)

[23] Migórski, S., Ogorzaly, J.: Dynamic history-dependent variational-hemivariational inequalities with applications to contact mechanics. Z. Angew. Math. Phys. 68, 15 (2017). https://doi.org/10.1007/s00033-016-0758-4

[24] Migórski, S., Zeng, S.: Hyperbolic hemivariational inequalities controlled by evolution equations with application to adhesive contact model. Nonlinear Anal. RWA 43, 121-143 (2018)

[25] Naniewicz, Z., Panagiotopoulos, P.D.: Mathematical Theory of Hemivariational Inequalities and Applications. Marcel Dekker Inc, New York (1995)

[26] Panagiotopoulos, P.D.: Hemivariational Inequalities, Applications in Mechanics and Engineering. Springer, Berlin (1993)

[27] Peng, Z.: Existence and regularity results for doubly nonlinear inclusions with nonmonotone perturbation. Nonlinear Anal. 115, 71-88 (2015)

[28] Sofonea, M., Han, W., Migórski, S.: Numerical analysis of history-dependent variational-hemivariational inequalities with applications to contact problems. Eur. J. Appl. Math. 26, 427-452 (2015)

[29] Sofonea, M., Matei, A.: History-dependent quasivariational inequalities arising in contact mechanics. Eur. J. Appl. Math. 22, 471-491 (2011)

[30] Sofonea, M., Matei, A.: Mathematical Models in Contact Mechanics. London Mathematical Society Lecture Note Series, vol. 398. Cambridge University Press, Cambridge (2012)

[31] Sofonea, M., Migórski, S.: A class of history-dependent variational-hemivariational inequalities. Nonlinear Differ. Equ. Appl. (2016). https://doi.org/10.1007/s00030-016-0391-0

[32] Sofonea, M., Migórski, S.: Variational-Hemivariational Inequalities with Applications. Monographs and Research Notes in Mathematics. Chapman \& Hall/CRC, Boca Raton (2018)

[33] Sofonea, M., Migórski, S., Ochal, A.: Two history-dependent contact problems, Chapter 14. In: Han, W., et al. (eds.) Advances in Variational and Hemivariational Inequalities: Theory, Numerical Analysis, and Applications. Advances in Mechanics and Mathematics Series, vol. 33, pp. 355-380. Springer, Cham (2015) 
[34] Zeng, B., Migórski, S.: Evolutionary subgradient inclusions with nonlinear weakly continuous operators and applications. Comput. Math. Appl. 75, 89-104 (2018)

[35] Zeng, S., Liu, Z., Migórski, S.: A class of fractional differential hemivariational inequalities with application to contact problem. Z. Angew. Math. Phys. 69, 36 (2018)

Stanisław Migórski

College of Sciences

Beibu Gulf University

Qinzhou 535000 Guangxi

People's Republic of China

e-mail: stanislaw.migorski@uj.edu.pl

Stanisław Migórski

Chair of Optimization and Control

Jagiellonian University in Krakow

ul. Lojasiewicza 6

30348 Kraków

Poland

Yunru Bai

Faculty of Mathematics and Computer Science

Jagiellonian University in Krakow

ul. Lojasiewicza 6

30348 Kraków

Poland

e-mail: angela-baivip@163.com

(Received: May 6, 2019; revised: June 19, 2019) 